\title{
Single-nucleon potential decomposition of the nuclear symmetry energy
}

\author{
Rong Chen, ${ }^{1}$ Bao-Jun Cai, ${ }^{1}$ Lie-Wen Chen ${ }^{*},{ }^{1,2}$ Bao-An Li, ${ }^{3}$ Xiao-Hua Li,,${ }^{1,4}$ and Chang Xu ${ }^{5}$ \\ ${ }^{1}$ Department of Physics, Shanghai Jiao Tong University, Shanghai 200240, China \\ ${ }^{2}$ Center of Theoretical Nuclear Physics, National Laboratory of Heavy Ion Accelerator, Lanzhou 730000, China \\ ${ }^{3}$ Department of Physics and Astronomy, Texas A\&M University-Commerce, Commerce, Texas 75429-3011, USA \\ ${ }^{4}$ School of Nuclear Science and Technology, University of South China, Hengyang, Hunan 421001, China \\ ${ }^{5}$ Department of Physics, Nanjing University, Nanjing 210008, China
}

(Dated: October 29, 2018)

\begin{abstract}
The nuclear symmetry energy $E_{\text {sym }}(\rho)$ and its density slope $L(\rho)$ can be decomposed analytically in terms of the single-nucleon potential in isospin asymmetric nuclear matter. Using three popular nuclear effective interaction models which have been extensively used in nuclear structure and reaction studies, namely, the isospin and momentum dependent MDI interaction model, the Skyrme Hartree-Fock approach and the Gogny Hartree-Fock approach, we analyze the contribution of different terms in the single-nucleon potential to the $E_{\text {sym }}(\rho)$ and $L(\rho)$. Our results show that the observed different density behaviors of $E_{\text {sym }}(\rho)$ for different interactions are essentially due to the variation of the symmetry potential $U_{s y m, 1}(\rho, k)$. Furthermore, we find that the contribution of the second-order symmetry potential $U_{\text {sym }, 2}(\rho, k)$ to the $L(\rho)$ generally cannot be neglected. Moreover, our results demonstrate that the magnitude of the $U_{s y m, 2}(\rho, k)$ is generally comparable with that of $U_{\text {sym }, 1}(\rho, k)$, indicating the second-order symmetry potential $U_{\text {sym }, 2}(\rho, k)$ may have significant corrections to the widely used Lane approximation to the single-nucleon potential in extremely neutron(proton)-rich nuclear matter.
\end{abstract}

PACS numbers: 21.65.Ef, 21.30.Fe, 21.60.Jz

\section{INTRODUCTION}

During the last decade, the nuclear symmetry energy $E_{\text {sym }}(\rho)$ that essentially characterizes the isospin dependent part of the equation of state (EOS) of asymmetric nuclear matter has attracted much attention from different fields due to its multifaceted influences in nuclear physics and astrophysics [1 $[$ [ 6 as well as some interesting issues regarding possible new physics beyond the standard model [7-10]. For example, the density slope $L$ of the symmetry energy at nuclear matter saturation density $\rho_{0}$ has been shown to be important in determining several critical quantities such as the size of neutronskin in heavy nuclei [1] 20], location of neutron dripline [21], core-crust transition density [3, 4, 12, 22 25] and gravitational binding energy [26] of neutron stars. The symmetry energy may also have significant influence on gravitational wave emission from compact stars 27 31]. Furthermore, knowledge on the symmetry energy might be useful to understand the non-Newtonian gravity proposed in grand unified theories and to constrain properties of the neutral weakly coupled light spin-1 gauge $U$-boson originated from supersymmetric extensions of the standard model [10, 32 35].

In recent years, a great deal of experimental and theoretical efforts have been devoted to determining the density dependence of the symmetry energy [ [ $[,[\underline{6}]$. Although significant progress has been made, large uncertainties on $E_{\text {sym }}(\rho)$ still exist even around nuclear matter saturation

*Corresponding author (email: lwchen@sjtu.edu.cn) density, e.g., while the value of $E_{\text {sym }}\left(\rho_{0}\right)$ is determined to be around $30 \pm 4 \mathrm{MeV}$, mostly from analyzing nuclear masses, the extracted density slope $L$ scatters in a very large range from about 20 to $115 \mathrm{MeV}$, depending on the observables and methods used in the studies 17, 36, 37] (See, e.g., Refs. 19, 38 41 for a review of recent progress). To reduce the uncertainties on the constraints of $E_{\text {sym }}\left(\rho_{0}\right)$ and $L$ is thus of critical importance and remains a big challenge in the community.

So far, information on $E_{\text {sym }}\left(\rho_{0}\right)$ and its density slope $L$ is essentially obtained from theoretical model analysis on the experimental data of heavy ion collisions 36, 37, 42 44], nuclear mass [45, 46], excitation energies of isobaric analog states 47, pygmy dipole resonance of neutronrich nuclei 48, 49], isovector giant dipole resonance of neutron-rich nuclei 50, 51], and neutron skin thickness [17, 19]. In these theoretical models, an energy density functional with a number of parameters is usually assumed apriori, and the model parameters are then obtained from fitting experimental data and the empirical values of some physical quantities. Information on $E_{\text {sym }}\left(\rho_{0}\right)$ and $L$ is then extracted based on the obtained model parameters. Since all the phenomena/observables are in someway at least indirectly and qualitatively related to the $E_{\text {sym }}\left(\rho_{0}\right)$ and $L$, it is very useful to directly decompose $E_{\text {sym }}\left(\rho_{0}\right)$ and $L$ in terms of some relevant parts of the commonly used underlying nuclear effective interaction [52]. This decomposition of the $E_{\text {sym }}\left(\rho_{0}\right)$ and $L$ provides an important and physically more transparent approach to extract information about isospin dependence of strong interaction in nuclear medium from experiments and understand why the predicted symmetry energy from various models is so uncertain [53]. 
In a recent work 38|, based on the Hugenholtz-Van Hove $(\mathrm{HVH})$ theorem [54, 55], it was indeed shown that both $E_{\text {sym }}\left(\rho_{0}\right)$ and $L$ are completely determined by the single-nucleon potential in asymmetric nuclear matter which can be extracted from the nucleon global optical model potentials. In that work, the Lane approximation [56] to the single-nucleon potential in asymmetric nuclear matter, i.e., $U_{n / p}(\rho, \delta, k) \approx U_{0}(\rho, k) \pm U_{\text {sym }, 1}(\rho, k) \delta$, has been assumed and also the momentum independent nucleon effective mass has been used, therefore, the higherorder effects such as the contributions from the secondorder symmetry potential $U_{s y m, 2}(\rho, k)$ and the momentum dependence of the nucleon effective mass which can also contribute to $L$ have been neglected. So far, to our best knowledge, there is not any empirical or experimental information or even any theoretical predictions on the second-order symmetry potential $U_{\text {sym }, 2}(\rho, k)$. It is thus interesting and important to estimate the $U_{\text {sym }, 2}(\rho, k)$ with some well-established theoretical models.

The main motivation of the present work is to evaluate the $U_{\text {sym }, 2}(\rho, k)$ and estimate its contribution to $L$ based on several popular nuclear effective interaction models which have been extensively used in nuclear structure and reaction studies. Our results indicate that, although the momentum dependence of the nucleon effective mass might not be important, the second-order symmetry potential $U_{\text {sym }, 2}(\rho, k)$ might have nonnegligible contribution to the $L$. Furthermore, we find that the magnitude of the $U_{\text {sym }, 2}(\rho, k)$ is generally comparable with that of $U_{\text {sym }, 1}(\rho, k)$, indicating the secondorder symmetry potential $U_{\text {sym }, 2}(\rho, k)$ may have significant corrections to the widely used Lane approximation $U_{n / p}(\rho, \delta, k) \approx U_{0}(\rho, k) \pm U_{\text {sym }, 1}(\rho, k) \delta$ for the singlenucleon potential in extremely neutron(proton)-rich nuclear matter, e.g., in neutron stars and the neutronskin region of heavy nuclei. These results imply that it is important to extract experimentally information on $U_{\text {sym }, 2}(\rho, k)$.

The paper is organized as follows. In Section III we briefly recall the definition of the symmetry energy and the symmetry potential in asymmetric nuclear matter, and then derive the explicit expressions for the singlenucleon potential decomposition of the symmetry energy and its density slope. The results and discussions are presented in Section III. A summary is then given in Section IV] For completeness, the theoretical models used in the present paper are briefly described in the Appendix.

\section{THEORETICAL FORMULISM}

\section{A. The symmetry energy and the symmetry potential in asymmetric nuclear matter}

Due to the exchange symmetry between protons and neutrons in nuclear matter when one neglects the Coulomb interaction and assumes the charge symmetry of nuclear forces, the EOS of isospin asymmetric nuclear matter, defined by its binding energy per nucleon, can be expanded as a power series of even-order terms in isospin asymmetry $\delta$ as

$$
E(\rho, \delta)=E_{0}(\rho)+E_{\mathrm{sym}}(\rho) \delta^{2}+O\left(\delta^{4}\right),
$$

where $\rho=\rho_{n}+\rho_{p}$ is the baryon density and $\delta=\left(\rho_{n}-\right.$ $\left.\rho_{p}\right) / \rho$ is the isospin asymmetry with $\rho_{n}$ and $\rho_{p}$ denoting the neutron and proton densities, respectively; $E_{0}(\rho)=$ $E(\rho, \delta=0)$ is the EOS of symmetric nuclear matter, and the nuclear symmetry energy is expressed as

$$
E_{\mathrm{sym}}(\rho)=\left.\frac{1}{2 !} \frac{\partial^{2} E(\rho, \delta)}{\partial \delta^{2}}\right|_{\delta=0} .
$$

The higher-order terms of $\delta$ in Eq. (11) are negligible, leading to the well-known empirical parabolic law for the EOS of asymmetric nuclear matter, which has been verified by all many-body theories to date, at least for densities up to moderate values (See, e.g., Ref. [6] ).

Around the nuclear matter saturation density $\rho_{0}$, the nuclear symmetry energy $E_{\text {sym }}(\rho)$ can be expanded as

$$
E_{\text {sym }}(\rho)=E_{\text {sym }}\left(\rho_{0}\right)+L \chi+O\left(\chi^{2}\right),
$$

where $\chi=\left(\rho-\rho_{0}\right) / 3 \rho_{0}$ is a dimensionless variable and $L=L\left(\rho_{0}\right)$ is the density slope parameter of the symmetry energy at $\rho_{0}$. More generally, the slope parameter of the symmetry energy at arbitrary density $\rho$ is defined as

$$
L(\rho)=3 \rho \frac{d E_{\mathrm{sym}}(\rho)}{d \rho} .
$$

The slope parameter $L$ at $\rho_{0}$ characterizes the density dependence of the nuclear symmetry energy around nuclear matter saturation density $\rho_{0}$, and thus carry important information on the properties of nuclear symmetry energy at both high and low densities.

The single-nucleon potential $U_{\tau}(\rho, \delta, k)$ (we assume $\tau=1$ for neutrons and -1 for protons in this work) in asymmetric nuclear matter generally depends on the baryon density $\rho$, the isospin asymmetry $\delta$ and the amplitude of the nucleon momentum $k$. Due to the isospin symmetry of nuclear interactions under the exchange of neutrons and protons, the single-nucleon potential $U_{\tau}(\rho, \delta, k)$ can be expanded as a power series of $\delta$ as 52 .

$$
\begin{aligned}
U_{\tau}(\rho, \delta, k)= & U_{0}(\rho, k)+\sum_{i=1,2, \cdots} U_{\text {sym }, i}(\rho, k)(\tau \delta)^{i} \\
= & U_{0}(\rho, k)+U_{\text {sym }, 1}(\rho, k)(\tau \delta) \\
& +U_{\text {sym }, 2}(\rho, k)(\tau \delta)^{2}+\cdots,
\end{aligned}
$$

where $U_{0}(\rho, k) \equiv U_{n}(\rho, 0, k)=U_{p}(\rho, 0, k)$ is the singlenucleon potential in symmetric nuclear matter and $U_{\text {sym }, i}(\rho, k)$ are expressed as

$$
\begin{aligned}
U_{\text {sym }, i}(\rho, k) & \left.\equiv \frac{1}{i !} \frac{\partial^{i} U_{n}(\rho, \delta, k)}{\partial \delta^{i}}\right|_{\delta=0} \\
& =\left.\frac{(-1)^{i}}{i !} \frac{\partial^{i} U_{p}(\rho, \delta, k)}{\partial \delta^{i}}\right|_{\delta=0},
\end{aligned}
$$


with $U_{\text {sym.1 }}(\rho, k)$ being the well-known nuclear symmetry potential [6] (where $U_{s y m, 1}$ is denoted by $U_{\text {sym }}$ ), and the higher-order term $U_{\text {sym, }}(\rho, k)$ being called as the secondorder nuclear symmetry potential here. Neglecting the higher-order terms $\left(\delta^{2}, \delta^{3}, \ldots\right)$ in Eq. (5) leads to the well-known Lane potential [56], i.e.,

$$
U_{\tau}(\rho, \delta, k) \approx U_{0}(\rho, k)+U_{s y m, 1}(\rho, k)(\tau \delta),
$$

which has been extensively used to approximate the single-nucleon potential $U_{\tau}(\rho, \delta, k)$ in asymmetric nuclear matter, and in this case the symmetry potential $U_{\text {sym }, 1}(\rho, k)$ can be obtained approximately by [6, 57]

$$
U_{s y m, 1}(\rho, k) \approx \frac{U_{n}(\rho, \delta, k)-U_{p}(\rho, \delta, k)}{2 \delta} .
$$

\section{B. Single-nucleon potential decomposition of the symmetry energy and its density slope}

According to the HVH theorem [54, 55], the chemical potentials of neutrons and protons in asymmetric nuclear matter with energy density $\varepsilon(\rho, \delta)=\rho E(\rho, \delta)$ can be expressed, respectively, as

$$
\begin{aligned}
t\left(k_{F_{n}}\right)+U_{n}\left(\rho, \delta, k_{F_{n}}\right) & =\frac{\partial \varepsilon(\rho, \delta)}{\partial \rho_{n}}, \\
t\left(k_{F_{p}}\right)+U_{p}\left(\rho, \delta, k_{F_{p}}\right) & =\frac{\partial \varepsilon(\rho, \delta)}{\partial \rho_{p}},
\end{aligned}
$$

where $t\left(k_{F_{\tau}}\right)=k_{F_{\tau}}^{2} / 2 m$ is the nucleon kinetic energy at Fermi momentum $k_{F_{\tau}}=k_{F}(1+\tau \delta)^{1 / 3}$ with $k_{F}=$ $\left(3 \pi^{2} \rho / 2\right)^{1 / 3}$ being the Fermi momentum in symmetric nuclear matter at density $\rho$. We would like to point out that the HVH theorem is independent of the detailed nature of the nucleon interactions used and has been strictly proven to be valid for any interacting self-bound infinite Fermi system [54, 55].

The right-hand side of Eq. (9) can be further written as

$$
\begin{aligned}
\frac{\partial \varepsilon(\rho, \delta)}{\partial \rho_{n}} & =\frac{\partial \varepsilon(\rho, \delta)}{\partial \rho} \frac{\partial \rho}{\partial \rho_{n}}+\frac{\partial \varepsilon(\rho, \delta)}{\partial \delta} \frac{\partial \delta}{\partial \rho_{n}} \\
& =\frac{\partial \varepsilon(\rho, \delta)}{\partial \rho}+\frac{1}{\rho} \frac{\partial \varepsilon(\rho, \delta)}{\partial \delta}-\frac{\partial \varepsilon(\rho, \delta)}{\partial \delta} \frac{\delta}{\rho}
\end{aligned}
$$

Similarly, the right-hand side of Eq. (10) can be expressed as

$$
\frac{\partial \varepsilon(\rho, \delta)}{\partial \rho_{p}}=\frac{\partial \varepsilon(\rho, \delta)}{\partial \rho}-\frac{1}{\rho} \frac{\partial \varepsilon(\rho, \delta)}{\partial \delta}-\frac{\partial \varepsilon(\rho, \delta)}{\partial \delta} \frac{\delta}{\rho} .
$$

Subtracting Eq. (12) from Eq. (11) and noting $\varepsilon(\rho, \delta)=$ $\rho E(\rho, \delta)$, we then obtain

$$
\begin{aligned}
& \frac{\partial \varepsilon(\rho, \delta)}{\partial \rho_{n}}-\frac{\partial \varepsilon(\rho, \delta)}{\partial \rho_{p}} \\
= & \frac{2}{\rho} \frac{\partial \varepsilon(\rho, \delta)}{\partial \delta}=2 \frac{\partial E(\rho, \delta)}{\partial \delta}
\end{aligned}
$$

while adding Eq. (11) and Eq. (12), we have

$$
\begin{aligned}
& \frac{\partial \varepsilon(\rho, \delta)}{\partial \rho_{n}}+\frac{\partial \varepsilon(\rho, \delta)}{\partial \rho_{p}} \\
= & 2 E(\rho, \delta)+2 \rho \frac{\partial E(\rho, \delta)}{\partial \rho}-2 \delta \frac{\partial E(\rho, \delta)}{\partial \delta} .
\end{aligned}
$$

On the one hand, substituting Eq. (11) into Eq. (13) and Eq. (14) respectively leads to following expressions, i.e.,

$$
\begin{aligned}
& t\left(k_{F_{n}}\right)-t\left(k_{F_{p}}\right)+U_{n}\left(\rho, \delta, k_{F_{n}}\right)-U_{p}\left(\rho, \delta, k_{F_{p}}\right) \\
= & 4 E_{\text {sym }}(\rho) \delta+\mathcal{O}\left(\delta^{3}\right)
\end{aligned}
$$

and

$$
\begin{aligned}
& t\left(k_{F_{n}}\right)+t\left(k_{F_{p}}\right)+U_{n}\left(\rho, \delta, k_{F_{n}}\right)+U_{p}\left(\rho, \delta, k_{F_{p}}\right) \\
= & 2 E_{0}(\rho)+2 \rho \frac{\partial E_{0}(\rho)}{\partial \rho} \\
+ & {\left[\frac{2}{3} L(\rho)-2 E_{\text {sym }}(\rho)\right] \delta^{2} } \\
+ & \mathcal{O}\left(\delta^{4}\right) .
\end{aligned}
$$

On the other hand, $t\left(k_{F_{\tau}}\right)$ and $U_{\tau}\left(\rho, \delta, k_{F_{\tau}}\right)$ can be expanded as a power series of $\delta$, respectively, as

$$
\begin{aligned}
& t\left(k_{F_{\tau}}\right)=t\left(k_{F}\right) \\
+ & \left.\frac{\partial t(k)}{\partial k}\right|_{k_{F}} \cdot \frac{1}{3} k_{F}(\tau \delta) \\
+ & \frac{1}{2}\left[\left.\frac{k_{F}^{2}}{9} \frac{\partial^{2} t(k)}{\partial k^{2}}\right|_{k_{F}}-\left.\frac{2 k_{F}}{9} \frac{\partial t(k)}{\partial k}\right|_{k_{F}}\right] \delta^{2} \\
+ & \mathcal{O}\left(\delta^{3}\right),
\end{aligned}
$$

and

$$
\begin{aligned}
& U_{\tau}\left(\rho, \delta, k_{F_{\tau}}\right)=U_{0}\left(\rho, k_{F}\right) \\
+ & {\left[\left.\frac{k_{F}}{3} \frac{\partial U_{0}(\rho, k)}{\partial k}\right|_{k_{F}}+U_{s y m, 1}\left(\rho, k_{F}\right)\right](\tau \delta) } \\
+ & {\left[\left.\frac{k_{F}}{3} \frac{\partial U_{s y m, 1}(\rho, k)}{\partial k}\right|_{k_{F}}+U_{s y m, 2}\left(\rho, k_{F}\right)\right] \delta^{2} } \\
+ & \frac{1}{2}\left[\left.\frac{k_{F}^{2}}{9} \frac{\partial^{2} U_{0}(\rho, k)}{\partial k^{2}}\right|_{k_{F}}-\left.\frac{2 k_{F}}{9} \frac{\partial U_{0}(\rho, k)}{\partial k}\right|_{k_{F}}\right] \delta^{2} \\
+ & \mathcal{O}\left(\delta^{3}\right) .
\end{aligned}
$$

Substituting Eqs. (17) and (18) into the left-hand sides of Eqs. (15) and (16), and comparing the coefficients of the 1st-order $\delta$ terms in both left and right hand sides, we then obtain

$$
E_{\text {sym }}(\rho)=\frac{1}{2} U_{\text {sym }, 1}\left(\rho, k_{F}\right)+\left.\frac{1}{6} \frac{\partial\left[t(k)+U_{0}(\rho, k)\right]}{\partial k}\right|_{k_{F}} \cdot k_{F},
$$

while comparing the coefficients of 2nd-order $\delta$ terms in both sides leads to the following expression

$$
\begin{aligned}
L(\rho) & =\frac{3}{2} U_{\text {sym }, 1}\left(\rho, k_{F}\right)+3 U_{\text {sym }, 2}\left(\rho, k_{F}\right) \\
& +\left.\frac{\partial U_{s y m, 1}}{\partial k}\right|_{k_{F}} \cdot k_{F}+\left.\frac{1}{6} \frac{\partial\left[t(k)+U_{0}(\rho, \delta)\right]}{\partial k}\right|_{k_{F}} \cdot k_{F} \\
& +\left.\frac{1}{6} \frac{\partial^{2}\left[t(k)+U_{0}(\rho, \delta)\right]}{\partial k^{2}}\right|_{k_{F}} \cdot k_{F}^{2} .
\end{aligned}
$$


It should be stressed that higher-order terms of the single-nucleon potential in Eq. (5) (i.e., $U_{s y m, 3}(\rho, k)$ and higher-order terms) have no contributions to $E_{\text {sym }}(\rho)$ and $L(\rho)$, and thus Eq. (19) and Eq. (20) are complete and exact, and are valid for arbitrary density $\rho$. Furthermore, Eq. (19) and Eq. (20) can be rewritten as

$$
\begin{aligned}
E_{\text {sym }}(\rho) & =\left.\frac{1}{3} \frac{\hbar^{2} k^{2}}{2 m_{0}^{*}}\right|_{k_{F}}+\frac{1}{2} U_{s y m, 1}\left(\rho, k_{F}\right) \\
L(\rho) & =\left.\frac{2}{3} \frac{\hbar^{2} k^{2}}{2 m_{0}^{*}}\right|_{k_{F}}-\left.\frac{1}{6}\left(\frac{\hbar^{2} k^{3}}{m_{0}^{* 2}} \frac{\partial m_{0}^{*}}{\partial k}\right)\right|_{k_{F}}+\frac{3}{2} U_{s y m, 1}\left(\rho, k_{F}\right)+\left.\frac{\partial U_{s y m, 1}}{\partial k}\right|_{k_{F}} \cdot k_{F}+3 U_{s y m, 2}\left(\rho, k_{F}\right)
\end{aligned}
$$

in terms of the nucleon effective mass $m_{0}^{*}(\rho, k)$ in symmetric nuclear matter which is generally dependent of the density $\rho$ and the nucleon momentum $k$, i.e.,

$$
m_{0}^{*}(\rho, k)=\frac{m}{1+\frac{m}{\hbar^{2} k} \frac{\partial U_{0}(\rho, k)}{\partial k}},
$$

due to the following relations

$$
\begin{aligned}
\left.\frac{\partial\left[t(k)+U_{0}(\rho, k)\right]}{\partial k}\right|_{k_{F}} & =\left.\frac{\hbar^{2} k}{m_{0}^{*}}\right|_{k_{F}}, \\
\left.\frac{\partial^{2}\left[t(k)+U_{0}(\rho, \delta)\right]}{\partial k^{2}}\right|_{k_{F}} & =\left.\frac{\hbar^{2}}{m_{0}^{*}}\right|_{k_{F}}-\left.\left(\frac{\hbar^{2} k}{m_{0}^{* 2}} \frac{\partial m_{0}^{*}}{\partial k}\right)\right|_{k_{F}} .
\end{aligned}
$$

For convenience, we can reexpress $E_{\text {sym }}(\rho)$ and $L(\rho)$, respectively, as

$$
\begin{aligned}
E_{\text {sym }}(\rho) & =E_{1}(\rho)+E_{2}(\rho) \\
L(\rho) & =L_{1}(\rho)+L_{2}(\rho)+L_{3}(\rho)+L_{4}(\rho)+L_{5}(\rho),
\end{aligned}
$$

with

$$
\begin{aligned}
& E_{1}(\rho)=\frac{1}{3} \frac{\hbar^{2} k_{F}^{2}}{2 m_{0}^{*}\left(\rho, k_{F}\right)} \\
& E_{2}(\rho)=\frac{1}{2} U_{\text {sym }, 1}\left(\rho, k_{F}\right) \\
& L_{1}(\rho)=\frac{2}{3} \frac{\hbar^{2} k_{F}^{2}}{2 m_{0}^{*}\left(\rho, k_{F}\right)} \\
& L_{2}(\rho)=-\left.\frac{1}{6} \frac{\hbar^{2} k_{F}^{3}}{m_{0}^{* 2}\left(\rho, k_{F}\right)} \frac{\partial m_{0}^{*}(\rho, k)}{\partial k}\right|_{k_{F}} \\
& L_{3}(\rho)=\frac{3}{2} U_{\text {sym }, 1}\left(\rho, k_{F}\right) \\
& L_{4}(\rho)=\left.\frac{\partial U_{\text {sym }, 1}(\rho, k)}{\partial k}\right|_{k_{F}} \cdot k_{F} \\
& L_{5}(\rho)=3 U_{\text {sym }, 2}\left(\rho, k_{F}\right) .
\end{aligned}
$$

In this way, one can see that $E_{1}(\rho)$ represents the kinetic energy part (including the effective mass contribution) of the symmetry energy while $E_{2}(\rho)$ is due to the symmetry potential contribution to the symmetry energy. Furthermore, $L_{1}(\rho), L_{2}(\rho), L_{3}(\rho), L_{4}(\rho)$, and $L_{5}(\rho)$ have respective physical meaning, namely, $L_{1}(\rho)$ represents the kinetic energy part (including the effective mass contribution) of the $L$ parameter, $L_{2}(\rho)$ is from the momentum dependence of the nucleon effective mass, $L_{3}(\rho)$ is due to the symmetry potential contribution, $L_{4}(\rho)$ comes from the momentum dependence of the symmetry potential, and $L_{5}(\rho)$ is from the second-order symmetry potential $U_{\text {sym, } 2}\left(\rho, k_{F}\right)$. In this way, the symmetry energy $E_{\text {sym }}(\rho)$ and its slope $L(\rho)$ have been decomposed in terms of $U_{0}(\rho, k), U_{\text {sym,1 }}(\rho, k), U_{\text {sym }, 2}(\rho, k)$ and/or their 1 st and 2nd-order partial derivatives with respect to $k$ and $\delta$. In particular, at nuclear matter saturation density $\rho_{0}, U_{0}\left(\rho_{0}, k\right), U_{\text {sym }, 1}\left(\rho_{0}, k\right)$ and $U_{s y m, 2}\left(\rho_{0}, k\right)$ (and thus $E_{\text {sym }}\left(\rho_{0}\right)$ and its slope parameter $\left.L\right)$ can be determined completely from the isospin and momentum dependent nucleon global optical potential, which can be directly extracted from nucleon-nucleus and $(\mathrm{p}, \mathrm{n})$ charge-exchange reactions (See, e.g., Refs. [38, 58, 59]).

For the single-nucleon potential decomposition of the slope parameter $L(\rho)$, if we use the Lane potential Eq. (17) and neglect the contributions from the momentum dependence of the nucleon effective mass, the $L(\rho)$ is then reduced to

$$
L(\rho)=L_{1}+L_{3}+L_{4},
$$

namely,

$$
\begin{aligned}
L(\rho) & =\left.\frac{2}{3} \frac{\hbar^{2} k^{2}}{2 m_{0}^{*}}\right|_{k_{F}}+\frac{3}{2} U_{s y m, 1}\left(\rho, k_{F}\right) \\
& +\left.\frac{\partial U_{s y m, 1}(\rho, k)}{\partial k}\right|_{k_{F}} \cdot k_{F},
\end{aligned}
$$

which has been used in previous work [38]. Although the Lane potential could be a good approximation in evaluating $U_{\text {sym,1 }}(\rho, k)$ as in Eq. (8] [6, 57], it would be interesting to see if the higher-order $U_{s y m, 2}\left(\rho, k_{F}\right)$ contribution to the $L(\rho)$ is significant or not. Using three popular nuclear effective interaction models, we will demonstrate in the following that the contribution of higher-order $U_{\text {sym }, 2}(\rho, k)$ term to $L(\rho)$ generally cannot be neglected.

\section{RESULTS AND DISCUSSIONS}

In the following, we analyze the single-nucleon potential decomposition of $E_{\text {sym }}(\rho)$ and $L(\rho)$ as well as the 
density and momentum dependence of $U_{\text {sym }, 1}(\rho, k)$ and $U_{\text {sym }, 2}(\rho, k)$ using three popular nuclear effective interaction models which have been extensively used in nuclear structure and reaction studies, namely, the isospin and momentum dependent MDI interaction model, the Skyrme Hartree-Fock approach, and the Gogny HartreeFock approach. One can find the details about these three models in the Appendix. A very useful feature of these models is that analytical expressions for many interesting physical quantities, such as the single-nucleon potential in asymmetric nuclear matter at zero temperature, can be obtained, and this makes our analysis and calculations physically transparent and very convenient.

For the MDI interaction, we use 3 parameter sets, i.e., $x=-1, x=0$ and $x=1$ 36], which give three different density dependence of the symmetry energy, namely, stiff one, moderate one, and soft one, respectively, and have been applied extensively in transport model simulations for heavy ion collisions. For the Skyrme interaction, we mainly use the famous SKM* 60 and SLy4 61] as well as the recently developed MSL0 [19]. In addition, a number of other Skyrme interactions are used for the singlenucleon potential decomposition of $E_{\text {sym }}(\rho)$ and $L(\rho)$ at $\rho_{0}$. For the Gogny interaction, we use the existing D1 [62], D1S [63], D1N 64], and D1M 65] which have been successfully applied in nuclear structure studies.

\section{A. Single-nucleon potential decomposition of $E_{\text {sym }}(\rho)$}

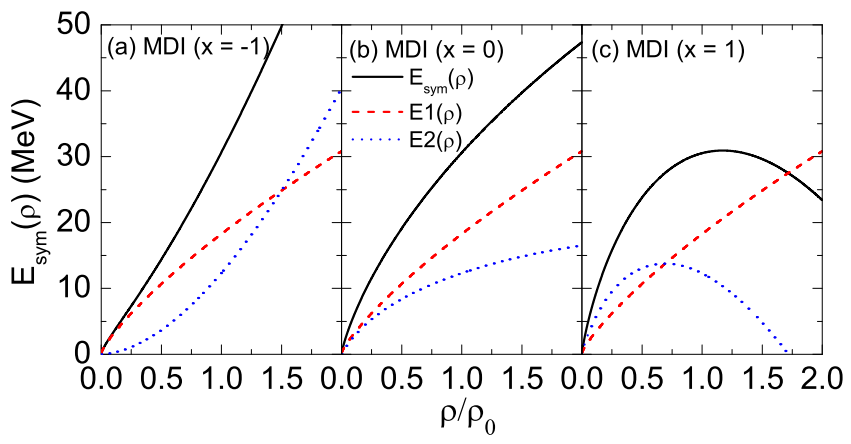

FIG. 1: (Color online) Density dependence of $E_{\text {sym }}(\rho)$, $E_{1}(\rho)=\frac{1}{3} \frac{\hbar^{2} k_{F}^{2}}{2 m_{0}^{*}\left(\rho, k_{F}\right)}$ and $E_{2}(\rho)=\frac{1}{2} U_{s y m, 1}\left(\rho, k_{F}\right)$ in the MDI interaction with $x=-1$ (a), 0 (b), and 1 (c).

In Fig. 1. Fig. 2 and Fig. 3, we plot the density dependence of $E_{\text {sym }}(\rho), E_{1}(\rho)$ and $E_{2}(\rho)$ in the MDI interaction model, the Skyrme-Hartree-Fock approach, and the Gogny-Hartree-Fock approach, respectively. One can see from Fig. 1 that for different $x$ values, the $E_{1}(\rho)$ displays the same density dependence while the $E_{2}(\rho)$ exhibits very different density behaviors, indicating that the different density dependences of $E_{\text {sym }}(\rho)$ for $x=-1,0$ and 1 are completely due to the different density dependence of $E_{2}(\rho)$, i.e., the symmetry potential $U_{s y m, 1}\left(\rho, k_{F}\right)$. The

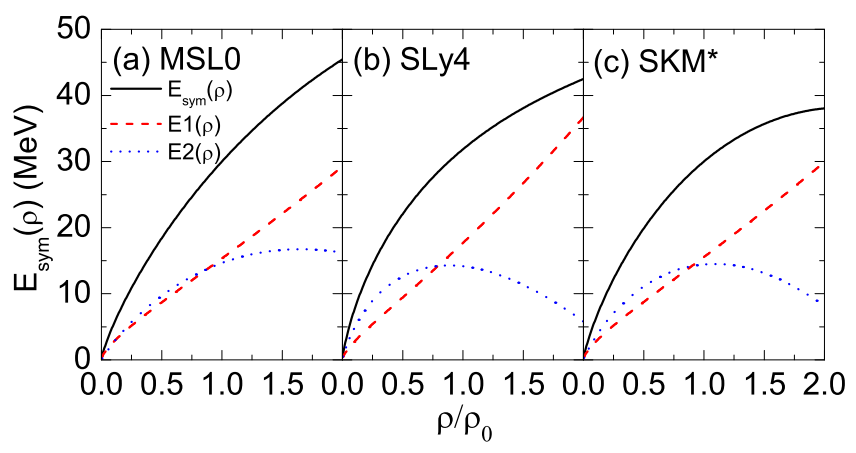

FIG. 2: (Color online) Same as Fig. 1 but in the Skyrme Hartree-Fock approach with MSL0 (a), SLy4 (b), and SKM* (c).

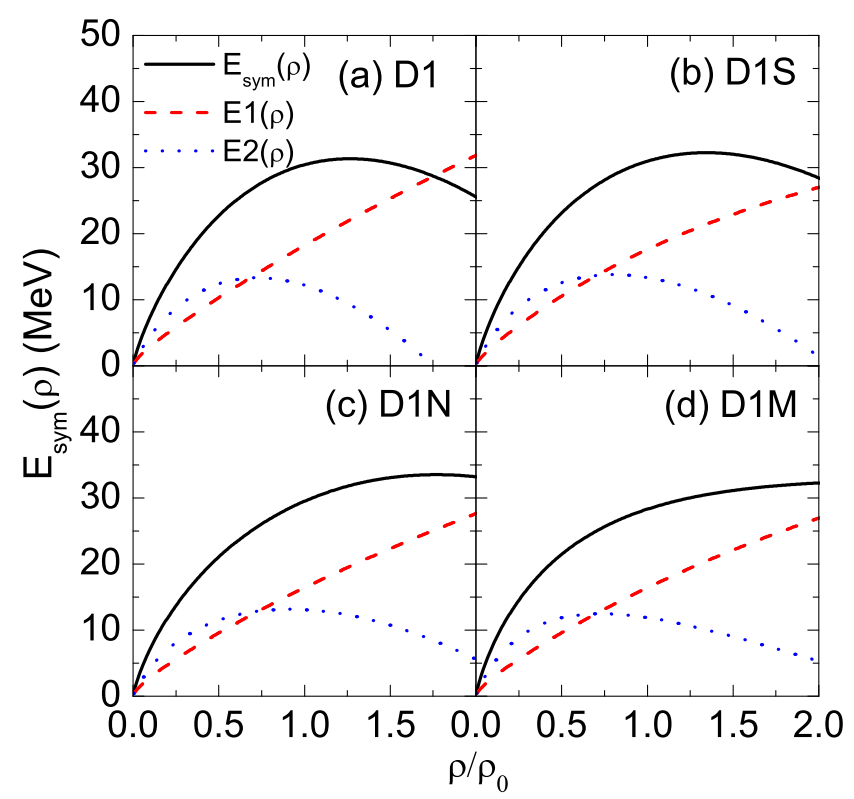

FIG. 3: (Color online) Same as Fig. 1 but in Gogny HartreeFock approach with D1 (a), D1S (b), D1N (c), and D1M (d).

similar behaviors can also be seen from Fig. 2 for the Skyrme-Hartree-Fock calculations and Fig. 3 for the Gogny-Hartree-Fock calculations.

Furthermore, one can see from Fig. 11. Fig. 2 and Fig. 3 that for all the interactions considered here, the $E_{1}(\rho)$ increases with the density and is always positive while $E_{2}(\rho)$ can increase or decrease with the density and even become negative at higher densities. These features can be understood since the $E_{1}(\rho)=\frac{1}{3} \frac{\hbar^{2} k_{F}^{2}}{2 m_{0}^{*}\left(\rho, k_{F}\right)}$ is determined uniquely by the single-nucleon potential $U_{0}(\rho, k)$ in symmetric nuclear matter for which reliable information about its density and momentum dependence has already been obtained from heavy-ion collisions, see, e.g., Ref. [2], albeit there is still some room for further improvements, particularly at high momenta/densities, and the nuclear effective interactions are usually constructed to describe reasonably $U_{0}(\rho, k)$, 
especially around $\rho_{0}$. However, on the contrary, the symmetry potential $U_{s y m, 1}\left(\rho, k_{F}\right)$, which mainly reflects the isospin dependence of the nuclear effective interaction in nuclear medium, is still not very well determined, especially at high densities and momenta. In fact, it has been identified as the key quantity responsible for the uncertain high density behavior of the symmetry energy as stressed in Ref. [6] (See also Refs. [52, 53]). These results show that the observed different density behaviors of $E_{\text {sym }}(\rho)$ for different interactions are essentially due to the variation of the symmetry potential $U_{s y m, 1}(\rho, k)$.

\section{B. Single-nucleon potential decomposition of $L(\rho)$}

In order to illustrate the single-nucleon potential decomposition of $L(\rho)$, we show in Fig. 4. Fig. [5] and Fig. 6] the density dependence of $L(\rho), L_{1}(\rho), L_{2}(\rho)$, $L_{3}(\rho), L_{4}(\rho)$ and $L_{5}(\rho)$ in the MDI interaction model, the Skyrme-Hartree-Fock approach, and the Gogny-HartreeFock approach, respectively. It is seen that the $L_{1}(\rho)$ displays the similar density dependence for all the interactions considered here, just like the $E_{1}(\rho)$ shown in Fig. 1] Fig. 2 and Fig. 3 because of $L_{1}(\rho)=2 E_{1}(\rho)$. The $L_{2}(\rho)$ is seen to contribute a small negative value to the $L(\rho)$, indicating that the momentum dependence of the nucleon effective mass is generally unimportant. In particular, one can see from Fig. 5 that $L_{2}(\rho)$ vanishes for the Skyrme-Hartree-Fock calculations due to the fact that the nucleon effective mass is momentum independent for the zero-range Skyrme interaction considered here. The $L_{3}(\rho)$ exhibits different density dependences for different interactions, reflecting the variation of $U_{\text {sym,1 }}\left(\rho, k_{F}\right)$ with density for different interactions. The $L_{4}(\rho)$ represents the contribution of the momentum dependence of the symmetry potential to the $L(\rho)$, and it displays different density dependence for different interactions and can be negative or positive, depending on the interaction used.

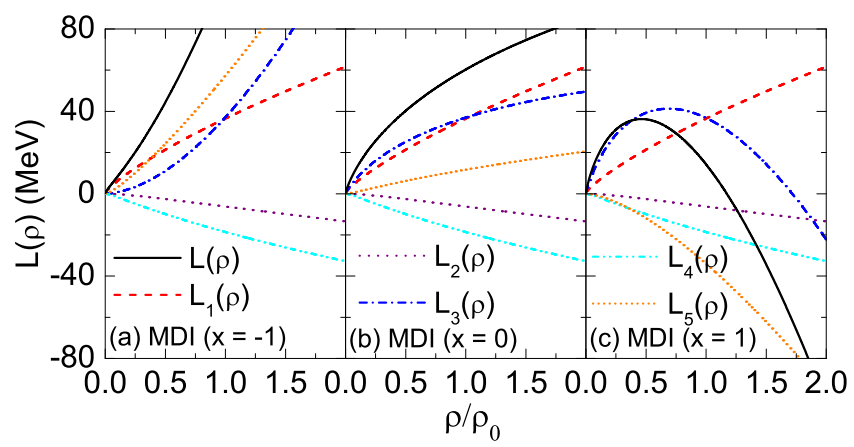

FIG. 4: (Color online) Density dependence of $L(\rho), L_{1}(\rho)=$ $\frac{2}{3} \frac{\hbar^{2} k_{F}^{2}}{2 m_{0}^{*}\left(\rho, k_{F}\right)}, \quad L_{2}(\rho)=-\left.\frac{1}{6} \frac{\hbar^{2} k_{F}^{3}}{m_{0}^{* 2}\left(\rho, k_{F}\right)} \frac{\partial m_{0}^{*}(\rho, k)}{\partial k}\right|_{k_{F}}, \quad L_{3}(\rho)=$ $\frac{3}{2} U_{\text {sym }, 1}\left(\rho, k_{F}\right), L_{4}(\rho)=\left.\frac{\partial U_{s y m, 1}(\rho, k)}{\partial k}\right|_{k_{F}} \cdot k_{F}$, and $L_{5}(\rho)=$ $3 U_{\text {sym }, 2}\left(\rho, k_{F}\right)$ in the MDI interaction with $x=-1$ (a), 0 (b), and 1 (c).

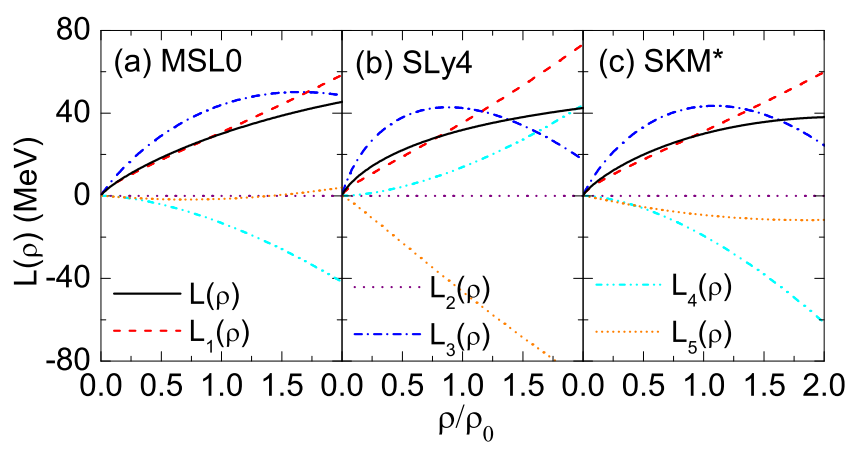

FIG. 5: (Color online) Same as Fig. 4 but in the Skyrme Hartree-Fock approach with MSL0 (a), SLy4 (b), and SKM* (c).

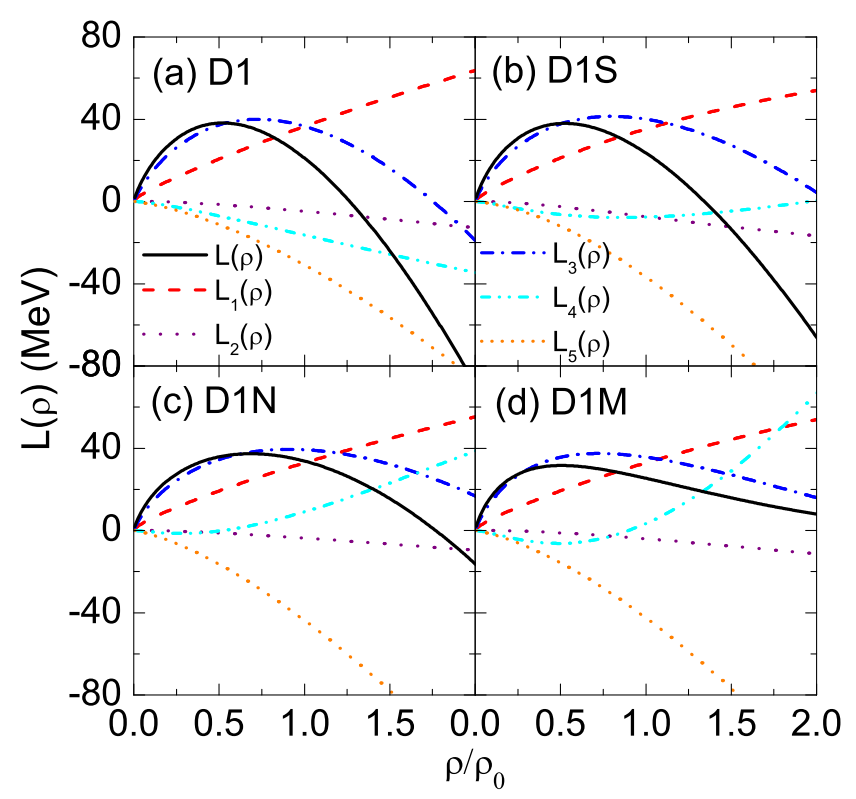

FIG. 6: (Color online) Same as Fig. 4 but in Gogny HartreeFock approach with D1 (a), D1S (b), D1N (c), and D1M (d).

It is particularly interesting to analyze the $L_{5}(\rho)$ since it reflects the higher-order $U_{s y m, 2}\left(\rho, k_{F}\right)$ contribution to the $L(\rho)$ and has been neglected in the previous work 38]. From Fig. 4, Fig. 5] and Fig. 6, it is surprising to see that the $L_{5}(\rho)$ may play an important role to determine the $L(\rho)$. In the MDI interaction with $x=-1$, the $L_{5}(\rho)$ is always positive and increases rapidly with density while the opposite behavior is observed for the MDI interaction with $x=1$. For the MDI interaction with $x=0$, the $L_{5}(\rho)$ is positive and moderately increases with density. For the Skyrme-Hartree-Fock calculations, the $L_{5}(\rho)$ can be positive or negative while it is always negative for the Gogny-Hartree-Fock calculations with D1, D1S, D1N, and D1M. These results indicate that generally the higher-order $U_{s y m, 2}\left(\rho, k_{F}\right)$ contribution to the $L(\rho)$ cannot be neglected and the Lane potential approximation to the single-nucleon potential 
in asymmetric nuclear matter may cause significant error for the determination of $L(\rho)$ from the single-nucleon potential decomposition.

Due to the special interest on the values of $E_{\text {sym }}(\rho)$ and $L(\rho)$ at $\rho_{0}$, we list in Table@ the values of the characteristic parameters $\rho_{0}, E_{0}\left(\rho_{0}\right), E_{\text {sym }}\left(\rho_{0}\right), E_{1}, E_{2}, L$, $L_{1}, L_{2}, L_{3}, L_{4}$ and $L_{5}$ at $\rho_{0}$ for the MDI interaction with $x=-1,0$ and 1 , the Gogny-Hartree-Fock predictions with D1, D1S, D1N, and D1M as well as the SkyrmeHartree-Fock predictions with 112 standard Skyrme interactions (In the table, the interactions in different models are in order according to the $L$ value and the corresponding reference for different interactions is included in the last column). For the MDI interaction model, one can see from Table 1 that the value of $E_{2}\left(\rho_{0}\right)$ is comparable with that of $E_{1}\left(\rho_{0}\right)$, and all the $E_{0}\left(\rho_{0}\right), E_{1}\left(\rho_{0}\right)$, and $E_{2}\left(\rho_{0}\right)$ are the same for different $x$ values due to the fact that the $U_{0}(\rho, k)$ and $U_{\text {sym }, 1}\left(\rho_{0}, k\right)$ are independent of the $x$ parameter by construction [36]. Because of the same reason, the values of $L_{1}, L_{2}, L_{3}, L_{4}$ are all independent of the $x$ parameter too. Therefore, for the MDI interaction, the $x$ dependence of the $L$ parameter is completely determined by the second-order symmetry potential $U_{\text {sym, } 2}\left(\rho, k_{F}\right)$. Depending on the value of the $x$ parameter, the $U_{\text {sym,2 }}\left(\rho, k_{F}\right)$ can be positive or negative. In particular, we have $L=L_{1}+L_{2}+L_{3}+L_{4}=48.5$ $\mathrm{MeV}$ if we assume $U_{s y m, 2}\left(\rho, k_{F}\right)=0$. Furthermore, it is seen that the $L_{2}$ contribution is relatively small compared with that of $L_{1}, L_{3}$, or $L_{4}$, indicating that the contribution due to the momentum dependence of the nucleon effective mass is unimportant, and this is consistent with the observation from Fig. 4.

For the Gogny interaction, the value of $L$ listed in Table 1 ranges from about $18 \mathrm{MeV}$ to $34 \mathrm{MeV}$. And similarly with the MDI interaction model, the $L_{2}$ is relatively small, ranging from about $-8 \mathrm{MeV}$ to $-3 \mathrm{MeV}$. It is interesting to see that the values of $L_{1}$ and $L_{3}$ from the Gogny interactions are quite similar with those of the MDI interaction model, and thus the difference of the $L$ parameter from different interactions in these two models is mainly due to the variation of the $L_{4}$ and $L_{5}$. Furthermore, for different Gogny interactions considered here, the value of $L_{4}$ can be positive or negative while the value of $L_{5}$ is always negative, and the $L_{5}$ contribution to the $L$ parameter usually is relatively important.

For the standard Skyrme interactions, we have $L_{2}=$ $0 \mathrm{MeV}$. For the 112 Skyrme interactions considered in Table 1 it is seen that the value of $L$ ranges from about $-50 \mathrm{MeV}$ to $160 \mathrm{MeV}, L_{1}$ from about $20 \mathrm{MeV}$ to $60 \mathrm{MeV}$, $L_{3}$ from about $4 \mathrm{MeV}$ to $74 \mathrm{MeV}, L_{4}$ from about -48 $\mathrm{MeV}$ to $36 \mathrm{MeV}$, and $L_{5}$ from about $-102 \mathrm{MeV}$ to 58 $\mathrm{MeV}$. Therefore, the different term contributions to the $L$ parameter can change a lot in the standard Skyrme interactions, especially for $L_{3}, L_{4}$, and $L_{5}$.

In order to see more clearly and intuitively the different term contributions to the $L$ parameter, we show in Fig. 7 the correlations of $L_{1}\left(\rho_{0}\right), L_{3}\left(\rho_{0}\right), L_{4}\left(\rho_{0}\right)$, and $L_{5}\left(\rho_{0}\right)$ with $L\left(\rho_{0}\right)$ for the MDI interaction with $x=-1$,

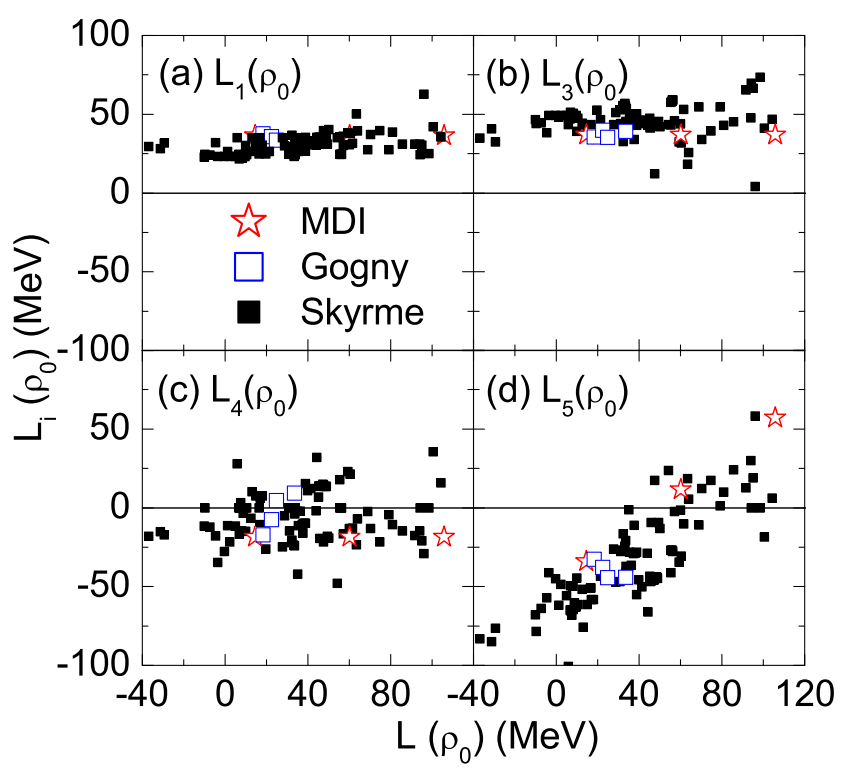

FIG. 7: (Color online) Correlations of $L_{1}\left(\rho_{0}\right)(\mathrm{a}), L_{3}\left(\rho_{0}\right)(\mathrm{b})$, $L_{4}\left(\rho_{0}\right)(\mathrm{c})$, and $L_{5}\left(\rho_{0}\right)(\mathrm{d})$ with $L\left(\rho_{0}\right)$ for the MDI interaction with $x=-1,0$ and 1, Gogny-Hartree-Fock predictions with D1, D1S, D1N and D1M as well as Skyrme-Hartree-Fock predictions with the 112 standard Skyrme interactions considered in Table \

0 and 1, the Gogny-Hartree-Fock predictions with D1, D1S, D1N, and D1M as well as the Skyrme-HartreeFock predictions with the 112 standard Skyrme interactions. One can see from Fig. 7 that the results from the MDI interaction model and the Gogny-Hartree-Fock calculations are essentially consistent with the systematics of the Skyrme-Hartree-Fock predictions. Based on these calculated results, we find that a statistical analysis can lead to $L_{1}\left(\rho_{0}\right) \approx 30 \pm 6.5 \mathrm{MeV}, L_{3}\left(\rho_{0}\right) \approx 46 \pm 9.5$ $\mathrm{MeV}, L_{4}\left(\rho_{0}\right) \approx-4 \pm 15 \mathrm{MeV}$, and $L_{5}\left(\rho_{0}\right) \approx-35 \pm 30$ $\mathrm{MeV}$. These results indicate that, within the standard Skyrme-Hartree-Fock energy density functional, $L_{1}\left(\rho_{0}\right)$ and $L_{3}\left(\rho_{0}\right)$ are relatively well constrained, and the main uncertainties are due to the $L_{4}\left(\rho_{0}\right)$ and $L_{5}\left(\rho_{0}\right)$ contributions. Furthermore, it is interesting to see from Fig. 7 that there exhibits an approximately linear correlation between $L_{5}\left(\rho_{0}\right)$ and $L\left(\rho_{0}\right)$. If we use the present empirical constraint $L\left(\rho_{0}\right)=60 \pm 30 \mathrm{MeV}$, then we find the $L_{5}\left(\rho_{0}\right)$ can vary from about $-66 \mathrm{MeV}$ to $24 \mathrm{MeV}$, i.e., the value of $U_{\text {sym,2 }}\left(\rho_{0}, k_{F}\right)$ can vary from about $-22 \mathrm{MeV}$ to $8 \mathrm{MeV}$.

\section{The symmetry potential $U_{s y m, 1}(\rho, k)$}

Shown in Fig. 8 is the momentum dependence of the $U_{\text {sym }, 1}(\rho, k)$ at $\rho=0.5 \rho_{0}, \rho_{0}$ and $2 \rho_{0}$ using the MDI interaction with $x=-1,0$, and 1. For comparison, we also include in Fig. 8 the corresponding results from several microscopic approaches, including the relativistic impulse approximation (RIA) [103, 104] us- 
TABLE I: The characteristic parameters $\rho_{0}\left(\mathrm{fm}^{-3}\right), E_{0}\left(\rho_{0}\right)(\mathrm{MeV}), E_{\text {sym }}\left(\rho_{0}\right)(\mathrm{MeV}), E_{1}(\mathrm{MeV}), E_{2}(\mathrm{MeV}), L(\mathrm{MeV}), L_{1}$ $(\mathrm{MeV}), L_{2}(\mathrm{MeV}), L_{3}(\mathrm{MeV}), L_{4}(\mathrm{MeV})$, and $L_{5}(\mathrm{MeV})$ at saturation density $\rho_{0}$ for the MDI interaction with $x=-1,0$ and 1, the Gogny-Hartree-Fock predictions with D1, D1S, D1N and D1M as well as the Skyrme-Hartree-Fock predictions with 112 standard Skyrme interactions. The interactions in different models are in order according to the $L$ value. The corresponding reference is included as the last column.

\begin{tabular}{|c|c|c|c|c|c|c|c|c|c|c|c|c|}
\hline Model & $\rho_{0}$ & $E_{0}\left(\rho_{0}\right)$ & $E_{\text {sym }}\left(\rho_{0}\right)$ & $E_{1}$ & $E_{2}$ & $\mathrm{~L}$ & $L_{1}$ & $L_{2}$ & $L_{3}$ & $L_{4}$ & $L_{5}$ & Ref. \\
\hline \multicolumn{13}{|l|}{ MDI } \\
\hline MDI $x=1$ & 0.160 & -16.2 & 30.5 & 18.2 & 12.3 & 14.6 & 36.4 & -6.2 & 36.9 & -18.6 & -33.9 & [36] \\
\hline MDI $x=0$ & 0.160 & -16.2 & 30.5 & 18.2 & 12.3 & 60.2 & 36.4 & -6.2 & 36.9 & -18.6 & 11.6 & [36] \\
\hline MDI $x=-1$ & 0.160 & -16.2 & 30.5 & 18.2 & 12.3 & 105.7 & 36.4 & -6.2 & 36.9 & -18.6 & 57.2 & [36] \\
\hline \multicolumn{13}{|l|}{ Gogny } \\
\hline D1 & 0.166 & -16.3 & 30.7 & 18.8 & 11.9 & 18.4 & 37.7 & -5.0 & 35.6 & -17.1 & -32.7 & [62] \\
\hline D1S & 0.163 & -16.0 & 31.1 & 17.9 & 13.3 & 22.4 & 35.7 & -7.6 & 39.8 & -7.5 & -37.9 & [63] \\
\hline D1M & 0.165 & -16.0 & 28.6 & 16.8 & 11.8 & 24.8 & 33.6 & -4.2 & 35.3 & 4.5 & -44.3 & [65] \\
\hline $\mathrm{D} 1 \mathrm{~N}$ & 0.161 & -16.0 & 29.6 & 16.5 & 13.1 & 33.6 & 33.0 & -3.7 & 39.2 & 9.2 & -44.2 & [64] \\
\hline \multicolumn{13}{|l|}{ Skyrme } \\
\hline Z-fit & 0.159 & -16.0 & 26.8 & 14.5 & 12.3 & -49.7 & 29.0 & 0 & 36.9 & -13.3 & -102.4 & [67] \\
\hline Esigma-fit & 0.163 & -16.0 & 26.4 & 14.8 & 11.6 & -36.9 & 29.6 & 0 & 34.9 & -18.1 & -83.2 & [67] \\
\hline E-fit & 0.159 & -16.1 & 27.7 & 14.1 & 13.6 & -31.3 & 28.2 & 0 & 40.7 & -15.2 & -84.9 & [67] \\
\hline Zsigma-fit & 0.163 & -15.9 & 26.7 & 15.9 & 10.8 & -29.4 & 31.8 & 0 & 32.4 & -17.1 & -76.5 & [67] \\
\hline SVII & 0.143 & -15.8 & 27.0 & 11.4 & 15.6 & -10.2 & 22.8 & 0 & 46.7 & -11.7 & -68.0 & [68] \\
\hline $\mathrm{SkSC} 4 \mathrm{o}$ & 0.161 & -15.9 & 27.0 & 12.3 & 14.7 & -9.7 & 24.6 & 0 & 44.0 & 0 & -78.3 & [69] \\
\hline SVI & 0.143 & -15.8 & 26.9 & 12.0 & 14.9 & -7.3 & 24.1 & 0 & 44.6 & -12.2 & -63.8 & [68] \\
\hline ZsigmaS-fit & 0.162 & -16.0 & 28.8 & 16.0 & 12.8 & -4.5 & 32.0 & 0 & 38.4 & -17.9 & -57.0 & [67] \\
\hline v070 & 0.158 & -15.8 & 28.0 & 11.6 & 16.4 & -3.5 & 23.1 & 0 & 49.3 & -34.7 & -41.2 & [70] \\
\hline v075 & 0.158 & -15.8 & 28.0 & 11.6 & 16.4 & -0.3 & 23.1 & 0 & 49.3 & -27.8 & -45.0 & [70] \\
\hline SI & 0.155 & -16.0 & 29.2 & 13.2 & 16.0 & 1.2 & 26.4 & 0 & 48.1 & -11.4 & -61.9 & [67] \\
\hline v080 & 0.157 & -15.8 & 28.0 & 11.6 & 16.4 & 2.2 & 23.1 & 0 & 49.3 & -21.7 & -48.5 & [70] \\
\hline v090 & 0.157 & -15.8 & 28.0 & 11.6 & 16.4 & 5.0 & 23.1 & 0 & 49.3 & -11.6 & -55.8 & [70] \\
\hline Skz4 & 0.160 & -16.0 & 32.0 & 17.5 & 14.5 & 5.8 & 35.1 & 0 & 43.4 & 27.9 & -100.6 & [71] \\
\hline SkSC15 & 0.161 & -15.9 & 28.0 & 12.3 & 15.7 & 6.7 & 24.6 & 0 & 47.0 & 0 & -65.0 & [69] \\
\hline BSk3 & 0.157 & -15.8 & 27.9 & 10.8 & 17.1 & 6.8 & 21.6 & 0 & 51.3 & -16.6 & -49.6 & [72] \\
\hline MSk3 & 0.158 & -15.8 & 28.0 & 12.2 & 15.8 & 7.0 & 24.3 & 0 & 47.5 & 0 & -64.8 & [73] \\
\hline v105 & 0.157 & -15.8 & 28.0 & 11.6 & 16.4 & 7.1 & 23.1 & 0 & 49.3 & 0 & -65.3 & [70] \\
\hline MSk4 & 0.157 & -15.8 & 28.0 & 11.6 & 16.4 & 7.2 & 23.1 & 0 & 49.3 & 0 & -65.2 & [73] \\
\hline BSk1 & 0.157 & -15.8 & 27.8 & 11.6 & 16.2 & 7.2 & 23.1 & 0 & 48.7 & 0 & -64.7 & [74] \\
\hline v110 & 0.157 & -15.8 & 28.0 & 11.6 & 16.4 & 7.5 & 23.1 & 0 & 49.3 & 3.2 & -68.1 & [70] \\
\hline MSk5 & 0.157 & -15.8 & 28.0 & 11.6 & 16.4 & 7.6 & 23.1 & 0 & 49.3 & 0 & -64.9 & [73] \\
\hline BSk2p & 0.157 & -15.8 & 28.0 & 11.6 & 16.4 & 7.8 & 23.2 & 0 & 49.3 & -14.9 & -49.7 & [75] \\
\hline BSk2 & 0.157 & -15.8 & 28.0 & 11.7 & 16.3 & 8.0 & 23.3 & 0 & 49.0 & -14.8 & -49.6 & [75] \\
\hline MSk8 & 0.158 & -15.8 & 27.9 & 11.0 & 16.9 & 8.3 & 22.1 & 0 & 50.6 & 0 & -64.5 & [76] \\
\hline v100 & 0.157 & -15.8 & 28.0 & 11.6 & 16.4 & 8.7 & 23.1 & 0 & 49.3 & -3.5 & -60.2 & [70] \\
\hline MSk7 & 0.158 & -15.8 & 27.9 & 11.6 & 16.4 & 9.4 & 23.1 & 0 & 49.1 & 0 & -62.9 & [77] \\
\hline MSk6 & 0.157 & -15.8 & 28.0 & 11.6 & 16.4 & 9.6 & 23.1 & 0 & 49.3 & 0 & -62.8 & [73] \\
\hline SIII & 0.145 & -15.9 & 28.2 & 15.1 & 13.1 & 9.9 & 30.2 & 0 & 39.2 & -14.8 & -44.7 & [78] \\
\hline MSk9 & 0.158 & -15.8 & 28.0 & 12.2 & 15.8 & 10.4 & 24.3 & 0 & 47.5 & 0.0 & -61.5 & [76] \\
\hline BSk4 & 0.157 & -15.8 & 28.0 & 13.2 & 14.8 & 12.5 & 26.4 & 0 & 44.4 & -6.5 & -51.7 & [72] \\
\hline Skz3 & 0.160 & -16.0 & 32.0 & 17.5 & 14.5 & 13.0 & 35.1 & 0 & 43.4 & 10.3 & -75.8 & [71] \\
\hline BSk8 & 0.159 & -15.8 & 28.0 & 15.3 & 12.7 & 14.9 & 30.6 & 0 & 38.2 & 7.5 & -61.4 & [79] \\
\hline Dutta & 0.162 & -16.0 & 26.6 & 12.4 & 14.2 & 16.5 & 24.8 & 0 & 42.7 & 0 & -51.0 & [80] \\
\hline Skz2 & 0.160 & -16.0 & 32.0 & 17.5 & 14.5 & 16.8 & 35.1 & 0 & 43.4 & -10.7 & -50.9 & [71] \\
\hline BSk6 & 0.157 & -15.8 & 28.0 & 15.2 & 12.8 & 16.8 & 30.4 & 0 & 38.4 & 6.2 & -58.2 & [72] \\
\hline BSk7 & 0.157 & -15.8 & 28.0 & 15.2 & 12.8 & 18.0 & 30.4 & 0 & 38.4 & 7.5 & -58.3 & [72] \\
\hline SKP & 0.163 & -16.0 & 30.0 & 12.4 & 17.6 & 19.6 & 24.8 & 0 & 52.7 & -26.1 & -31.9 & [81] \\
\hline BSk5 & 0.157 & -15.8 & 28.7 & 13.2 & 15.5 & 21.4 & 26.4 & 0 & 46.5 & -8.0 & -43.5 & [72] \\
\hline Skz1 & 0.160 & -16.0 & 32.0 & 17.5 & 14.5 & 27.7 & 35.1 & 0 & 43.4 & -24.7 & -26.1 & [71] \\
\hline
\end{tabular}


TABLE I (Continued.)

\begin{tabular}{|c|c|c|c|c|c|c|c|c|c|c|c|c|}
\hline Model & $\rho_{0}$ & $E_{0}\left(\rho_{0}\right)$ & $E_{\text {sym }}\left(\rho_{0}\right)$ & $E_{1}$ & $E_{2}$ & $\mathrm{~L}$ & $L_{1}$ & $L_{2}$ & $L_{3}$ & $L_{4}$ & $L_{5}$ & Ref. \\
\hline SIIIs & 0.148 & -16.1 & 32.0 & 15.0 & 17.0 & 28.7 & 29.9 & 0 & 51.0 & -5.0 & -47.3 & [68] \\
\hline SKT6 & 0.161 & -16.0 & 30.0 & 12.3 & 17.6 & 30.9 & 24.7 & 0 & 52.9 & 0 & -46.7 & [82] \\
\hline SKT7 & 0.161 & -15.9 & 29.5 & 14.8 & 14.7 & 31.1 & 29.6 & 0 & 44.2 & -14.8 & -27.8 & [82] \\
\hline SKXm & 0.159 & -16.0 & 31.2 & 12.7 & 18.5 & 32.1 & 25.3 & 0 & 55.6 & -22.0 & -26.9 & [81] \\
\hline RATP & 0.160 & -16.0 & 29.2 & 18.4 & 10.9 & 32.4 & 36.8 & 0 & 32.6 & -20.5 & -16.5 & [83] \\
\hline SkSC14 & 0.161 & -15.9 & 30.0 & 12.3 & 17.7 & 33.1 & 24.6 & 0 & 53.0 & 0 & -44.5 & [69] \\
\hline SKX & 0.155 & -16.1 & 31.1 & 12.1 & 19.0 & 33.2 & 24.3 & 0 & 56.9 & -23.7 & -24.3 & [81] \\
\hline MSk2 & 0.157 & -15.8 & 30.0 & 11.6 & 18.4 & 33.4 & 23.1 & 0 & 55.3 & 0.0 & -45.1 & [73] \\
\hline SKXce & 0.155 & -15.9 & 30.1 & 12.0 & 18.2 & 33.5 & 23.9 & 0 & 54.5 & -24.2 & -20.8 & [81] \\
\hline BSk15 & 0.159 & -16.0 & 30.0 & 15.3 & 14.7 & 33.6 & 30.6 & 0.0 & 44.2 & -3.9 & -37.2 & [85] \\
\hline SKT8 & 0.161 & -15.9 & 29.9 & 14.8 & 15.1 & 33.7 & 29.6 & 0 & 45.4 & 0 & -41.3 & [82] \\
\hline SKT9 & 0.160 & -15.9 & 29.8 & 14.8 & 15.0 & 33.7 & 29.5 & 0 & 45.0 & 0 & -40.8 & [82] \\
\hline MSk1 & 0.157 & -15.8 & 30.0 & 12.2 & 17.8 & 33.9 & 24.3 & 0 & 53.5 & 0 & -43.9 & [73] \\
\hline BSk16 & 0.159 & -16.1 & 30.0 & 15.3 & 14.7 & 34.9 & 30.5 & 0.0 & 44.2 & -2.1 & -37.8 & [89] \\
\hline Skz0 & 0.160 & -16.0 & 32.0 & 17.5 & 14.5 & 35.1 & 35.1 & 0 & 43.4 & -42.1 & -1.3 & [71] \\
\hline Skyrme1p & 0.155 & -16.0 & 29.4 & 13.2 & 16.1 & 35.3 & 26.4 & 0 & 48.4 & -11.4 & -28.1 & [84] \\
\hline BSk17 & 0.159 & -16.1 & 30.0 & 15.3 & 14.7 & 36.3 & 30.5 & 0.0 & 44.2 & -2.1 & -36.4 & [90] \\
\hline BSk10 & 0.159 & -15.9 & 30.0 & 13.3 & 16.7 & 37.2 & 26.6 & 0 & 50.1 & -10.5 & -29.0 & [86] \\
\hline SGII & 0.158 & -15.6 & 26.8 & 15.5 & 11.3 & 37.6 & 31.0 & 0 & 33.9 & -16.1 & -11.2 & [87] \\
\hline BSk12 & 0.159 & -15.9 & 30.0 & 13.3 & 16.7 & 38.0 & 26.5 & 0 & 50.2 & -9.9 & -28.8 & [86] \\
\hline BSk11 & 0.159 & -15.9 & 30.0 & 13.3 & 16.7 & 38.4 & 26.5 & 0 & 50.2 & -9.8 & -28.6 & [86] \\
\hline SLy10 & 0.156 & -15.9 & 32.0 & 17.6 & 14.3 & 38.7 & 35.3 & 0 & 43.0 & 15.4 & -55.0 & [88] \\
\hline BSk13 & 0.159 & -15.9 & 30.0 & 13.3 & 16.7 & 38.8 & 26.5 & 0 & 50.2 & -9.7 & -28.2 & [86] \\
\hline BSk9 & 0.159 & -15.9 & 30.0 & 15.3 & 14.7 & 39.9 & 30.6 & 0 & 44.2 & 10.8 & -45.7 & [79] \\
\hline $\mathrm{KDE}$ & 0.164 & -16.0 & 32.0 & 16.5 & 15.4 & 41.4 & 33.1 & 0 & 46.3 & 12.3 & -50.2 & [91] \\
\hline BSk14 & 0.159 & -15.9 & 30.0 & 15.3 & 14.7 & 43.9 & 30.5 & 0.0 & 44.2 & -2.0 & -28.8 & [92] \\
\hline SLy230a & 0.160 & -16.0 & 32.0 & 17.6 & 14.4 & 44.3 & 35.2 & 0 & 43.1 & 32.0 & -66.1 & 61] \\
\hline KDE0 & 0.161 & -16.1 & 33.0 & 17.2 & 15.8 & 45.2 & 34.4 & 0 & 47.4 & 6.9 & -43.5 & [91] \\
\hline SLy8 & 0.160 & -16.0 & 31.4 & 17.7 & 13.8 & 45.3 & 35.3 & 0 & 41.3 & 13.7 & -45.1 & [88] \\
\hline SLy4 & 0.160 & -16.0 & 31.8 & 17.6 & 14.2 & 45.4 & 35.3 & 0 & 42.5 & 13.8 & -46.2 & [61] \\
\hline SLy0 & 0.161 & -16.0 & 31.5 & 17.6 & 13.8 & 45.4 & 35.3 & 0 & 41.5 & 13.6 & -45.0 & [88] \\
\hline SLy3 & 0.160 & -16.0 & 32.1 & 17.7 & 14.4 & 45.5 & 35.3 & 0 & 43.2 & 13.8 & -46.8 & [88] \\
\hline SKMs & 0.160 & -15.8 & 30.0 & 15.6 & 14.4 & 45.8 & 31.2 & 0 & 43.3 & -19.4 & -9.3 & [60] \\
\hline SLy230b & 0.160 & -16.0 & 32.0 & 17.6 & 14.4 & 46.0 & 35.3 & 0 & 43.1 & 13.9 & -46.4 & [124] \\
\hline SLy7 & 0.158 & -15.9 & 32.0 & 17.7 & 14.3 & 47.2 & 35.4 & 0 & 42.8 & 15.0 & -46.0 & [61] \\
\hline SLy6 & 0.159 & -15.9 & 32.0 & 17.7 & 14.2 & 47.4 & 35.4 & 0 & 42.7 & 14.6 & -45.3 & [61] \\
\hline $\mathrm{SKb}$ & 0.155 & -16.0 & 23.9 & 19.8 & 4.1 & 47.5 & 39.6 & 0 & 12.3 & -21.6 & 17.3 & [87] \\
\hline SLy5 & 0.160 & -16.0 & 32.0 & 17.6 & 14.4 & 48.3 & 35.3 & 0 & 43.1 & 13.7 & -43.8 & [61] \\
\hline SLy2 & 0.160 & -15.9 & 32.3 & 17.6 & 14.7 & 48.8 & 35.2 & 0 & 44.0 & 13.6 & -44.1 & [88] \\
\hline SLy1 & 0.160 & -16.0 & 32.5 & 17.6 & 14.9 & 48.8 & 35.2 & 0 & 44.7 & 13.7 & -44.8 & [88] \\
\hline SKM & 0.160 & -15.8 & 30.7 & 15.6 & 15.2 & 49.3 & 31.2 & 0 & 45.5 & -18.0 & -9.3 & [93] \\
\hline SII & 0.148 & -16.0 & 34.2 & 20.1 & 14.0 & 50.0 & 40.3 & 0 & 42.0 & -19.2 & -13.1 & [66] \\
\hline Skzm1 & 0.160 & -16.0 & 32.0 & 17.5 & 14.5 & 54.1 & 35.1 & 0 & 43.4 & -47.9 & 23.6 & [71] \\
\hline SKT3 & 0.161 & -15.9 & 31.5 & 12.3 & 19.2 & 55.3 & 24.7 & 0 & 57.5 & 0 & -26.8 & [82] \\
\hline SLy9 & 0.151 & -15.8 & 32.1 & 17.8 & 14.4 & 55.4 & 35.5 & 0 & 43.2 & 17.9 & -41.1 & [88] \\
\hline SKT3s & 0.160 & -16.0 & 31.7 & 12.3 & 19.4 & 55.9 & 24.6 & 0 & 58.2 & 0 & -26.9 & [82] \\
\hline SKT1s & 0.160 & -16.0 & 32.0 & 12.3 & 19.7 & 56.1 & 24.6 & 0 & 59.2 & 0 & -27.7 & [82] \\
\hline SKT2 & 0.161 & -15.9 & 32.0 & 12.3 & 19.7 & 56.2 & 24.7 & 0 & 59.0 & 0 & -27.5 & [82] \\
\hline SKT1 & 0.161 & -16.0 & 32.0 & 12.3 & 19.7 & 56.2 & 24.7 & 0 & 59.1 & 0 & -27.5 & [82] \\
\hline MSkA & 0.153 & -16.0 & 30.3 & 15.1 & 15.3 & 57.2 & 30.1 & 0 & 45.9 & -16.4 & -2.4 & [94] \\
\hline SkI6 & 0.159 & -15.9 & 29.9 & 19.1 & 10.8 & 59.2 & 38.2 & 0 & 32.4 & 23.1 & -34.4 & [95] \\
\hline MSL0 & 0.160 & -16.0 & 30.0 & 15.4 & 14.6 & 60.0 & 30.7 & 0 & 43.9 & -13.2 & -1.5 & [19] \\
\hline SkI4 & 0.160 & -15.9 & 29.5 & 18.9 & 10.6 & 60.4 & 37.8 & 0 & 31.7 & 21.4 & -30.6 & [96] \\
\hline LNS & 0.175 & -15.3 & 33.4 & 15.8 & 17.7 & 61.5 & 31.5 & 0 & 53.0 & -12.8 & -10.2 & [97] \\
\hline
\end{tabular}


TABLE I (Continued.)

\begin{tabular}{lcccccccccccc}
\hline \hline Model & $\rho_{0}$ & $E_{0}\left(\rho_{0}\right)$ & $E_{\text {sym }}\left(\rho_{0}\right)$ & $E_{1}$ & $E_{2}$ & $\mathrm{~L}$ & $L_{1}$ & $L_{2}$ & $L_{3}$ & $L_{4}$ & $L_{5}$ & Ref. \\
\hline SIV & 0.151 & -16.0 & 31.2 & 25.1 & 6.1 & 63.5 & 50.2 & 0 & 18.4 & -23.5 & 18.5 & {$[78]$} \\
SGI & 0.154 & -15.9 & 28.3 & 19.7 & 8.6 & 63.9 & 39.5 & 0 & 25.8 & -7.0 & 5.6 & {$[87]$} \\
SKOs & 0.160 & -15.8 & 31.9 & 13.7 & 18.2 & 68.9 & 27.4 & 0 & 54.7 & -2.4 & -10.8 & {$[98]$} \\
SkMP & 0.157 & -15.6 & 29.9 & 18.6 & 11.3 & 70.3 & 37.1 & 0 & 34.0 & -13.1 & 12.3 & {$[99]$} \\
Ska & 0.155 & -16.0 & 32.9 & 19.8 & 13.1 & 74.6 & 39.6 & 0 & 39.4 & -21.6 & 17.3 & {$[100]$} \\
SKO & 0.160 & -15.8 & 32.0 & 13.7 & 18.2 & 79.1 & 27.5 & 0 & 54.7 & -4.3 & 1.3 & {$[98]$} \\
SKYT & 0.148 & -15.4 & 33.7 & 19.3 & 14.3 & 80.8 & 38.7 & 0 & 43.0 & -10.8 & 9.9 & {$[101]$} \\
Rsigma-fit & 0.158 & -15.6 & 30.6 & 15.5 & 15.0 & 85.7 & 31.1 & 0.0 & 45.1 & -14.6 & 24.1 & {$[67]$} \\
SK272 & 0.155 & -16.3 & 37.4 & 15.6 & 21.8 & 91.7 & 31.2 & 0 & 65.4 & -17.6 & 12.6 & {$[102]$} \\
Gsigma-fit & 0.158 & -15.6 & 31.4 & 15.5 & 15.9 & 94.0 & 31.0 & 0.0 & 47.6 & -14.6 & 30.0 & {$[67]$} \\
SKT4 & 0.159 & -16.0 & 35.5 & 12.2 & 23.2 & 94.1 & 24.5 & 0 & 69.7 & 0 & 0 \\
SK255 & 0.157 & -16.3 & 37.4 & 15.2 & 22.2 & 95.1 & 30.5 & 0 & 66.5 & -20.9 & 19.0 & {$[102]$} \\
SV & 0.155 & -16.0 & 32.8 & 31.4 & 1.4 & 96.1 & 62.8 & 0 & 4.2 & -29.1 & 58.2 & {$[67]$} \\
SKT5 & 0.164 & -16.0 & 37.0 & 12.5 & 24.5 & 98.5 & 25.0 & 0 & 73.6 & 0 & 0 \\
SkI3 & 0.158 & -16.0 & 34.8 & 21.1 & 13.8 & 100.5 & 42.1 & 0 & 41.3 & 35.5 & -18.4 & {$[82]$} \\
SkI2 & 0.158 & -15.8 & 33.4 & 17.7 & 15.6 & 104.3 & 35.5 & 0 & 46.9 & 15.7 & 6.2 & {$[96]$} \\
SkI5 & 0.156 & -15.8 & 36.6 & 20.8 & 15.8 & 129.3 & 41.7 & 0 & 47.4 & 35.0 & 5.2 & {$[96]$} \\
SkI1 & 0.160 & -16.0 & 37.5 & 17.7 & 19.8 & 161.1 & 35.5 & 0 & 59.3 & 14.2 & 52.0 & {$[96]$} \\
\hline \hline
\end{tabular}

ing the empirical nucleon-nucleon scattering amplitude determined by Murdock and Horowitz (MH) [105] with isospin-dependent and isospin-independent Pauli blocking corrections as well as by McNeil, Ray, and Wallace (MRW) [106], the relativistic Dirac-Brueckner-HartreeFock (DBHF) theory [107], and the non-relativistic Brueckner-Hartree-Fock (BHF) theory with and without the three-body force (TBF) rearrangement contribution [108]. For these microscopic results, one can see that they are all consistent with each other around and below $\rho_{0}$ although there still exist larger uncertainties at higher density of $\rho=2 \rho_{0}$. It is interesting to see that the momentum dependence of the $U_{\text {sym, }}(\rho, k)$ from the MDI interaction with $x=0$ are in good agreement with the results from the microscopic approaches. It should be noted that the momentum dependence of the $U_{s y m, 1}(\rho, k)$ at $\rho_{0}$ is the same for $x=-1,0$, and 1 since $U_{s y m, 1}(\rho, k)$ is independent of the $x$ parameter at $\rho_{0}$ by construction as mentioned previously.

Similarly as in Fig. 8, we plot in Fig. 9 and Fig. 10 the the momentum dependence of the $U_{s y m, 1}(\rho, k)$ at $\rho=0.5 \rho_{0}, \rho_{0}$ and $2 \rho_{0}$ using the Skyrme Hartree-Fock approach with MSL0, SLy4 and SKM*, and the Gogny Hartree-Fock approach with D1, D1S, D1N and D1M, respectively. On the one hand, it is seen from Fig. 9 that, for $0<p<700 \mathrm{MeV} / \mathrm{c}$, the results from the MSL0 and $\mathrm{SKM}^{*}$ interactions agree well with those from microscopic approaches while the results from the SLy4 interaction seem to display large deviation from the microscopic results, especially at higher nucleon momenta. On the other hand, one can see from Fig. 10 that at $\rho=0.5 \rho_{0}$ and $\rho_{0}$, the results from D1 interaction are consistent with those of the microscopic calculations while the results from D1S, D1N and D1M exhibit large devia-

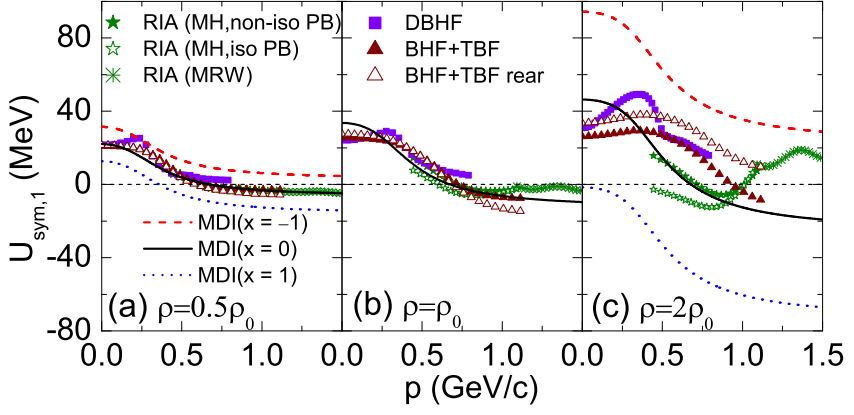

FIG. 8: (Color online) Momentum dependence of the $U_{\text {sym }, 1}(\rho, k)$ at $\rho=0.5 \rho_{0}(\mathrm{a}), \rho_{0}$ (b) and $2 \rho_{0}$ (c) using the MDI interaction with $x=-1,0$, and 1 . The corresponding results from several microscopic approaches are also included for comparison (See the text for details).

tion from the the microscopic calculations except at low nucleon momenta $(p \lesssim 300 \mathrm{MeV} / \mathrm{c})$. At $\rho=2 \rho_{0}$, the Gogny Hartree-Fock calculations display different results from the microscopic ones and the strong model dependence appears.

Overall, one can see from Fig. 8, Fig. 9 and Fig. 10 that the momentum dependence of the $U_{\text {sym,1 }}$ varies from one interaction to another for the MDI interaction model, the Skyrme Hartree-Fock approach and the Gogny Hartree-Fock approach. For the MDI interaction with $x=-1,0$, and 1 , the Gogny interaction with D1, and the Skyrme interaction with MSL0 and SKM*, the value of $U_{\text {sym,1 }}$ can be negative at higher momentum while for the Gogny interaction with D1S, D1N, D1M and the Skyrme interaction with SLy4, the value of $U_{s y m, 1}$ is positive at higher momentum. 


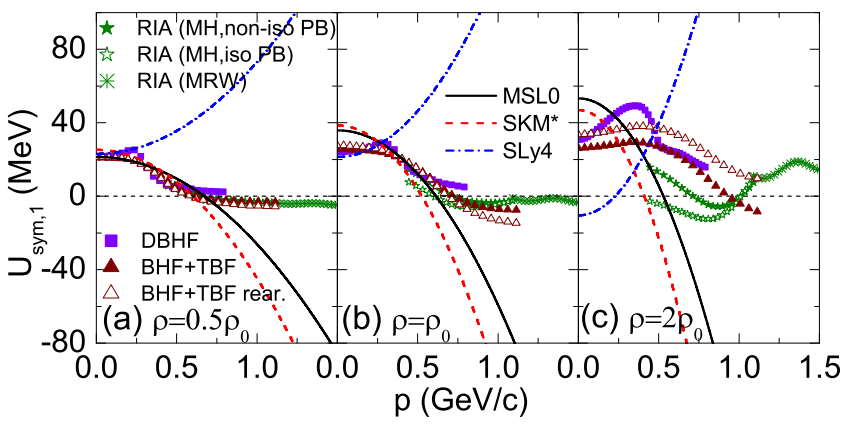

FIG. 9: (Color online) Same as Fig. 8 but for the Skyrme Hartree-Fock approach with MSL0, SLy4, and SKM*.

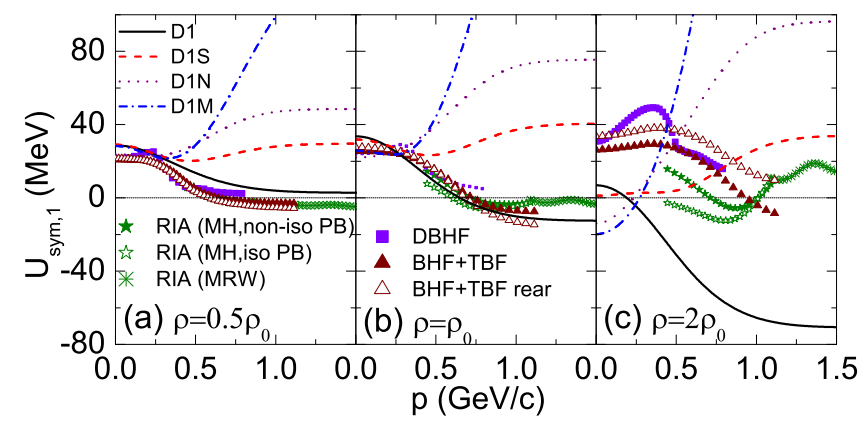

FIG. 10: (Color online) Same as Fig. 8 but for Gogny HartreeFock approach with D1, D1S, D1N, and D1M.

\section{The second-order symmetry potential $U_{\text {sym }, 2}(\rho, k)$}

As for $U_{\text {sym }, 2}$, to our best knowledge, there is not any experimental/empirical information or theoretical predictions so far. Fig. 11 and Fig. 12 show the momentum dependence of $U_{\text {sym, } 2}$ at $0.5 \rho_{0}, \rho_{0}$ and $2 \rho_{0}$ in the MDI interaction model and the Gogny Hartree-Fock approach, respectively. Since $U_{\text {sym }, 2}$ is independent of the nucleon momentum in the Skyrme Hartree-Fock approach, we only show here its density dependence in Fig. 13. From Fig. 11] and Fig. 12 it is interesting to see that for all interactions in the MDI interaction model and the Gogny Hartree-Fock approach at $\rho=0.5 \rho_{0}, \rho_{0}$ and $2 \rho_{0}, U_{\text {sym }, 2}$ firstly decreases with nucleon momentum and then essentially saturates when the nucleon momentum is larger than about $500 \mathrm{MeV} / \mathrm{c}$. Especially, the results from the MDI interaction with $x=1$ seem to be in quantitative agreement with those from the Gogny Hartree-Fock approach. Furthermore, one can see from Fig. 11 and Fig. 12 that the magnitude of $U_{\text {sym, } 2}$ increases with the density, and this is also true for the Skyrme HartreeFock approach as shown in Fig. 13. Another interesting feature is that for the MDI interaction model and the Skyrme Hartree-Fock approach, $U_{\text {sym, } 2}$ can be either negative or positive while it is always negative for the Gogny Hartree-Fock approach with the interactions considered here. Therefore, any experimental constraints about $U_{\text {sym,2 }}$ will be very useful and important for constraining the theoretical models.

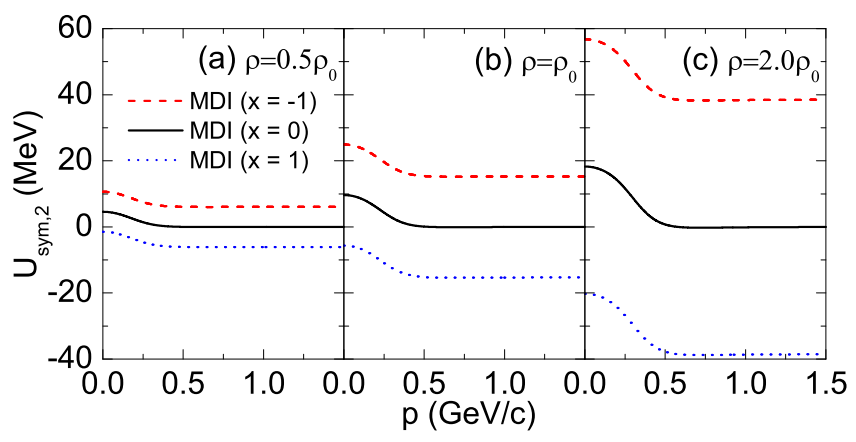

FIG. 11: (Color online) Momentum dependence of the $U_{\text {sym }, 2}(\rho, k)$ at $\rho=0.5 \rho_{0}(\mathrm{a}), \rho_{0}(\mathrm{~b})$ and $2 \rho_{0}(\mathrm{c})$ using the MDI interaction with $x=-1,0$, and 1 .

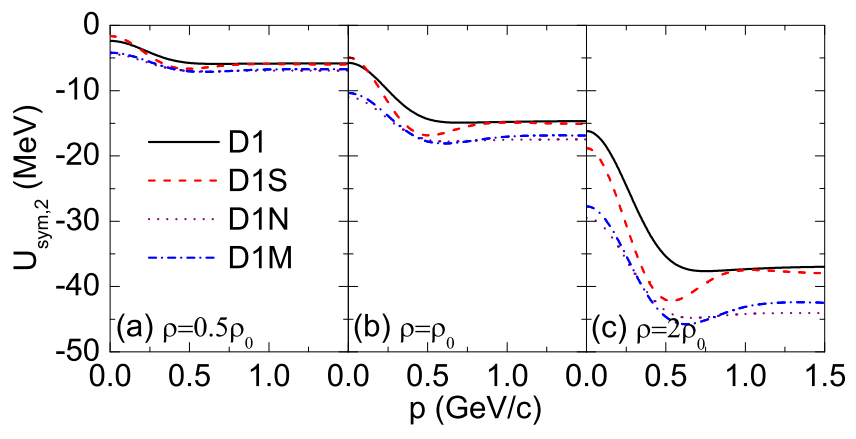

FIG. 12: (Color online) Same as Fig. 11 but for Gogny Hartree-Fock approach with D1, D1S, D1N, and D1M.

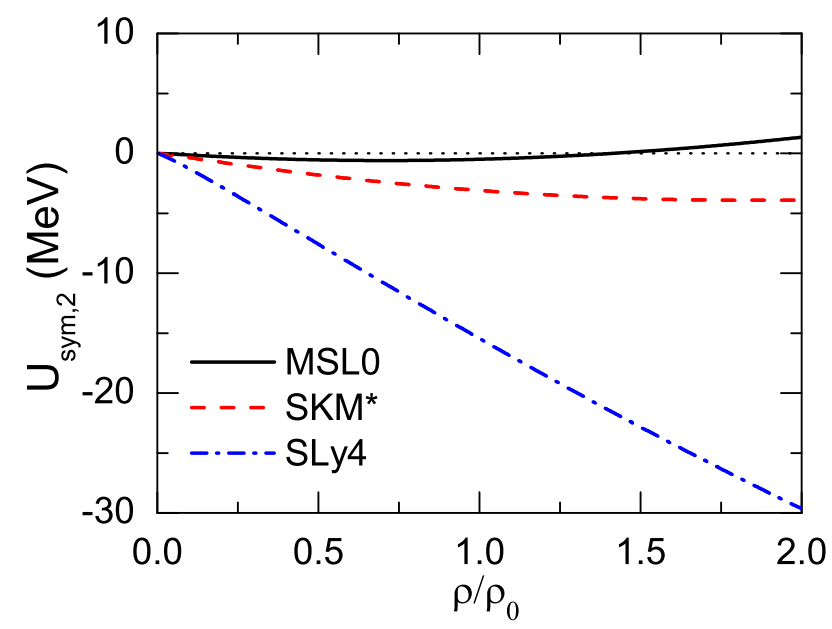

FIG. 13: (Color online) Density dependence of $U_{\text {sym }, 2}$ for the Skyrme Hartree-Fock approach with MSL0, SLy4, and SKM*.

Comparing Fig. 8 with Fig. 11 for the MDI interaction model, Fig. 9] with Fig. 13 for the Skyrme Hartree-Fock approach, and Fig. 10] with Fig. 12 for the Gogny HartreeFock approach, one can see that, at fixed density and 
momentum, the magnitude of $U_{s y m, 2}$ is generally comparable with that of $U_{s y m, 1}$. So, in Eq. (5)), compared with $U_{\text {sym }, 1}(\rho, k) \delta$, the contribution from $U_{\text {sym, } 2}(\rho, k) \delta^{2}$ could be negligible only if $\delta$ is small $(\delta \ll 1)$. Therefore, we conclude from the present model calculations that, when $\delta$ is small $(\delta \ll 1)$, the Lane potential might be a good approximation to the single-nucleon potential $U_{n / p}(\rho, \delta, p)$. However, the contributions of $U_{\text {sym,2 }}(\rho, k)$ might not be simply neglected when $\delta$ is close to 1 .

\section{SUMMARY}

Using the Hugenholtz-Van Hove theorem, we have explicitly and analytically expressed the symmetry energy $E_{\text {sym }}(\rho)$ and its density slope $L(\rho)$ in terms of the single-nucleon potential in asymmetric nuclear matter that might be extracted from experiments. We have carefully checked the contributions of each decomposed term, i.e., $E_{1}(\rho)$ and $E_{2}(\rho)$ for $E_{\text {sym }}(\rho)$, and $L_{1}(\rho), L_{2}(\rho)$, $L_{3}(\rho), L_{4}(\rho)$ and $L_{5}(\rho)$ for $L(\rho)$ by using three popular phenomenological nuclear interaction models in nuclear structure and reaction studies, namely, the isospin and momentum dependent MDI model, the Skyrme HartreeFock approach, and the Gogny Hartree-Fock approach.

Our results have indicated that the $E_{2}(\rho)$ due to the symmetry potential $U_{\text {sym, }}\left(\rho, k_{F}\right)$ is comparable with the $E_{1}(\rho)$ which describes the kinetic part including the nucleon effective mass contribution and the observed different density behaviors of $E_{\text {sym }}(\rho)$ for different interactions are essentially due to the variation of the symmetry potential $U_{s y m, 1}(\rho, k)$. For the density slope parameter $L(\rho)$, interestingly, we have found that, although the term $L_{2}$ due to the momentum dependence of the nucleon effective mass might not have significant contributions. the term $L_{5}$, which is from the second-order symmetry potential $U_{\text {sym,2 }}\left(\rho, k_{F}\right)$, generally cannot be negligible.

By analyzing the density and momentum dependence of $U_{\text {sym,1 }}(\rho, k)$ and $U_{\text {sym, } 2}(\rho, k)$ for the three nuclear effective interaction models, we have demonstrated that the magnitude of the second-order symmetry potential $U_{\text {sym }, 2}(\rho, k)$ is generally comparable with that of the symmetry potential $U_{s y m, 1}(\rho, k)$ and thus the Lane potential $U_{n / p}(\rho, \delta, k) \approx U_{0}(\rho, k) \pm U_{\text {sym }, 1}(\rho, k) \delta$ could be a good approximation to the single-nucleon potential only if the isospin asymmetry $\delta$ is small $(\delta \ll 1)$. However, $U_{\text {sym }, 2}(\rho, k)$ might not be neglected in describing the single-nucleon potential $U_{n / p}(\rho, \delta, k)$ in extremely neutron(proton)-rich nuclear matter, e.g., in neutron stars and the neutron-skin region of heavy nuclei, where the value of $\delta$ could be very large (close to 1 ).

While the momentum dependence of $U_{0}(\rho, k)$ and $U_{\text {sym,1 }}(\rho, k)$ has been extensively investigated and relatively well constrained from the measured nucleon optical model potentials, heavy ion collisions experiments, and the microscopic calculations, especially around and below nuclear matter saturation density, knowledge on $U_{\text {sym }, 2}(\rho, k)$ is still very poorly known. The results on
$U_{\text {sym }, 2}(\rho, k)$ presented here from the three phenomenological models have indicated large model dependence. Therefore, to constrain $U_{\text {sym }, 2}(\rho, k)$ from experiments or microscopic calculations (e.g., BHF and DBHF) based on nucleon-nucleon interactions derived from scattering phase shifts is crucial for a complete and more precise description for $U_{n / p}(\rho, \delta, k)$, and thus for $E_{\text {sym }}(\rho)$ and $L(\rho)$. Experimentally, information on the momentum dependence of $U_{\text {sym,2 }}\left(\rho_{0}, k\right)$ can be in principle obtained from the isospin dependent nucleon optical model potentials. On the other hand, all analyses in the present work are based on the non-relativistic models, it will be thus interesting to see how our results change in relativistic covariant energy-density functionals, such as the relativistic mean field models. These studies are in progress.

\section{ACKNOWLEDGMENTS}

This work was supported in part by the National Natural Science Foundation of China under Grant Nos. 10735010, 10775068, 10805026, 10975097, 11135011 and 11175085, Shanghai Rising-Star Program under grant No. 11QH1401100, "Shu Guang" project supported by Shanghai Municipal Education Commission and Shanghai Education Development Foundation, the Program for Professor of Special Appointment (Eastern Scholar) at Shanghai Institutions of Higher Learning, the National Basic Research Program of China (973 Program) under Contract No. 2007CB815004, the US National Science Foundation grant PHY-0757839, the Texas Coordinating Board of Higher Education grant No.003565-00042007, the National Aeronautics and Space Administration under grant NNX11AC41G issued through the Science Mission Directorate, and the Research Fund of Doctoral Point (RFDP), No. 20070284016.

\section{Appendix: Models for nuclear effective interactions}

For completeness, we briefly introduce in this Appendix the nuclear interaction models used in this work and also present some important expressions. These models include the isospin- and momentum-dependent MDI interaction, the Hartree-Fock approach based on Skyrme interactions, and the Hartree-Fock approach based on the finite-range Gogny interactions. These models have been extensively used in nuclear structure studies and transport model simulations for heavy ion collisions.

\section{Isospin- and momentum-dependent MDI interaction}

The isospin- and momentum-dependent MDI interaction is a phenomenological effective interaction based on a modified finite range Gogny interaction [36, 109]. In the 
MDI interaction, the potential energy density $\varepsilon_{\text {pot }}(\rho, \delta)$ of an asymmetric nuclear matter at total density $\rho$ and isospin asymmetry $\delta$ is given by

$$
\varepsilon_{p o t}(\rho, \delta)=\frac{A_{u}(x) \rho_{n} \rho_{p}}{\rho_{0}}+\frac{A_{l}(x)}{2 \rho_{0}}\left(\rho_{n}^{2}+\rho_{p}^{2}\right)+\frac{B}{\sigma+1} \frac{\rho^{\sigma+1}}{\rho_{0}^{\sigma}}\left(1-x \delta^{2}\right)+\frac{1}{\rho_{0}} \sum_{\tau, \tau^{\prime}} C_{\tau, \tau^{\prime}} \iint d \boldsymbol{p} d \boldsymbol{p}^{\prime} \frac{f_{\tau}(p) f_{\tau^{\prime}}\left(p^{\prime}\right)}{1+\left(\boldsymbol{p}-\boldsymbol{p}^{\prime}\right)^{2} / \Lambda^{2}}
$$

where $A_{u}(x)=-95.98-x \frac{2 B}{\sigma+1}(\mathrm{MeV}), A_{l}(x)=-120.57+$ $x \frac{2 B}{\sigma+1}(\mathrm{MeV}), B=106.35(\mathrm{MeV}), \sigma=4 / 3, C_{\tau, \tau}=$ $-11.70(\mathrm{MeV}), C_{\tau,-\tau}=-103.40(\mathrm{MeV})$, and $\Lambda=$ $\hbar\left(3 \pi^{2} \rho_{0} / 2\right)^{1 / 3}$ are obtained from fitting the momentum dependence of single-nucleon potential to that predicted by the Gogny Hartree-Fock and/or the BruecknerHartree-Fock calculations, the saturation properties of symmetric nuclear matter and the symmetry energy of $30.5 \mathrm{MeV}$ at nuclear matter saturation density $\rho_{0}=0.16$ $\mathrm{fm}^{-3}$. The incompressibility for cold symmetric nuclear matter at saturation density $\rho_{0}$ is set to be $K_{0}=211$ $\mathrm{MeV}$. The $x$ parameter in the MDI interaction is introduced to vary the density dependence of the nuclear symmetry energy while keeping other properties of the nuclear equation of state fixed [36], and it can be adjusted to mimic the predictions of microscopic and/or phenomeno- logical many-body theories on the density dependence of nuclear matter symmetry energy. We would like to point out that the MDI interaction has been extensively used in the transport model for studying isospin effects in intermediate energy heavy-ion collisions induced by neutron-rich nuclei 36, 110 117], the study of the thermal properties of asymmetric nuclear matter [118, 119], and the compact star physics 24, 120, 121. In particular, the isospin diffusion data from NSCL/MSU have constrained the value of $x$ to between 0 and -1 for nuclear matter densities less than about $1.2 \rho_{0}[\underline{36}]$.

In mean-field approximation, the single-nucleon potential for a nucleon with momentum $p$ and isospin $\tau$ in asymmetric nuclear matter can be expressed as 109, 122, 123]

$$
\begin{aligned}
& U_{\tau}(\rho, \delta, p)=A_{u}(x) \frac{\rho_{-\tau}}{\rho_{0}}+A_{l}(x) \frac{\rho_{\tau}}{\rho_{0}}+B\left(\frac{\rho}{\rho_{0}}\right)^{\sigma}\left(1-x \delta^{2}\right)-4 \tau x \frac{B}{\sigma+1} \frac{\rho^{\sigma-1}}{\rho_{0}^{\sigma}} \delta \rho_{-\tau} \\
+ & \frac{2 C_{\tau, \tau}}{\rho_{0}} \frac{2}{h^{3}} \pi \Lambda^{3}\left[\frac{p_{F_{\tau}}^{2}+\Lambda^{2}-p^{2}}{2 p \Lambda} \ln \frac{\left(p+p_{F_{\tau}}\right)^{2}+\Lambda^{2}}{\left(p-p_{F_{\tau}}\right)^{2}+\Lambda^{2}}+\frac{2 p_{F_{\tau}}}{\Lambda}-2 \arctan \frac{p+p_{F_{\tau}}}{\Lambda}+2 \arctan \frac{p-p_{F_{\tau}}}{\Lambda}\right] \\
+ & \frac{2 C_{\tau,-\tau}}{\rho_{0}} \frac{2}{h^{3}} \pi \Lambda^{3}\left[\frac{p_{F_{-\tau}}^{2}+\Lambda^{2}-p^{2}}{2 p \Lambda} \ln \frac{\left(p+p_{F_{-\tau}}\right)^{2}+\Lambda^{2}}{\left(p-p_{F_{-\tau}}\right)^{2}+\Lambda^{2}}+\frac{2 p_{F_{-\tau}}}{\Lambda}-2 \arctan \frac{p+p_{F_{-\tau}}}{\Lambda}+2 \arctan \frac{p-p_{F_{-\tau}}}{\Lambda}\right],
\end{aligned}
$$

where $\rho_{\tau}=\rho(1+\tau \delta) / 2$ and $p_{F_{\tau}}=\hbar\left(3 \pi^{2} \rho_{\tau}\right)^{1 / 3}$.

\section{Skyrme Hartree-Fock approach}

For the Skyrme interaction, we use the standard form 124 that has been shown to be very successful in describing the structure of finite nuclei. Neglecting the spin-orbit interaction term which is irrelevant in nuclear matter calculations considered here, the nuclear effective interaction in the standard Skyrme interaction is taken to have a zero-range, density- and momentum-dependent form [124], i.e.,

$$
\begin{aligned}
V_{12}^{\text {Skyrme }}\left(\boldsymbol{r}_{1}, \boldsymbol{r}_{2}\right) & =t_{0}\left(1+x_{0} P_{\sigma}\right) \delta(\boldsymbol{r})+\frac{1}{2} t_{1}\left(1+x_{1} P_{\sigma}\right)\left[\boldsymbol{P}^{\prime 2} \delta(\boldsymbol{r})+\delta(\boldsymbol{r}) \boldsymbol{P}^{2}\right]+t_{2}\left(1+x_{2} P_{\sigma}\right) \boldsymbol{P}^{\prime} \cdot \delta(\boldsymbol{r}) \boldsymbol{P} \\
& +\frac{1}{6} t_{3}\left(1+x_{3} P_{\sigma}\right)[\rho(\boldsymbol{R})]^{\alpha} \delta(\boldsymbol{r}),
\end{aligned}
$$

where $\boldsymbol{r}=\boldsymbol{r}_{1}-\boldsymbol{r}_{2}, \boldsymbol{R}=\left(\boldsymbol{r}_{1}+\boldsymbol{r}_{2}\right) / 2, P_{\sigma}$ is spin exchange operator, $\boldsymbol{P}=\frac{1}{2 i}\left(\boldsymbol{\nabla}_{1}-\boldsymbol{\nabla}_{2}\right)$ is relative momentum operator acting on the right and $\boldsymbol{P}^{\prime}$ is its conjugate which acts on the left. The $t_{0}, x_{0}, t_{1}, x_{1}, t_{2}, x_{2}, t_{3}, x_{3}$ and $\alpha$ are the 9 Skyrme interaction parameters determined from fitting the binding energies, charge radii, and other properties of a 
large number of nuclei in the periodic table. In the Skyrme Hartree-Fock approach, the single-nucleon potential in asymmetric nuclear matter is given by [124]

$$
\begin{aligned}
U_{\tau}(\rho, \delta, k) & =\frac{k^{2}}{8} \rho\left[t_{1}\left(2+x_{1}\right)+t_{2}\left(2+x_{2}\right)\right]+\frac{k^{2}}{8} \rho_{\tau}\left[t_{2}\left(1+2 x_{2}\right)-t_{1}\left(1+2 x_{1}\right)\right]+\frac{1}{2} t_{0}\left[\left(2+x_{0}\right) \rho-\left(2 x_{0}+1\right) \rho_{\tau}\right] \\
& +\frac{1}{12} t_{3} \rho^{\alpha}\left[\left(2+x_{3}\right) \rho-\left(2 x_{3}+1\right) \rho_{\tau}\right]+\frac{\alpha}{24} t_{3} \rho^{\alpha-1}\left[\left(2+x_{3}\right) \rho^{2}-\left(2 x_{3}+1\right)\left(\rho_{n}^{2}+\rho_{p}^{2}\right)\right] \\
& +\frac{1}{8}\left[t_{1}\left(2+x_{1}\right)+t_{2}\left(2+x_{2}\right)\right]\left(\frac{p_{F_{n}}^{5}}{5 \pi^{2} \hbar^{5}}+\frac{p_{F_{p}}^{5}}{5 \pi^{2} \hbar^{5}}\right)+\frac{1}{8}\left[t_{2}\left(2 x_{2}+1\right)-t_{1}\left(2 x_{1}+1\right)\right] \frac{p_{F_{\tau}}^{5}}{5 \pi^{2} \hbar^{5}}
\end{aligned}
$$

\section{Gogny interaction}

Gogny interaction has been proved to be very successful in describing not only nuclear structure but also nuclear matter [62, 125]. Compared with the Skyrme interaction which only contains $\delta$ forces, Gogny interaction is featured by two finite range terms plus one $\delta$ force that can well mimic the nucleon-nucleon effective interaction. Neglecting the spin-orbit interaction term, the conventional Gogny interaction is given by [62, 125]

$$
V_{12}^{G o g n y}\left(\boldsymbol{r}_{1}, \boldsymbol{r}_{2}\right)=\sum_{i=1}^{2}\left(W_{i}+B_{i} P_{\sigma}-H_{i} P_{\tau}-M_{i} P_{\sigma} P_{\tau}\right) e^{-\boldsymbol{r}^{2} / \mu_{i}^{2}}+t_{0}\left(1+x_{0} P_{\sigma}\right)[\rho(\boldsymbol{R})]^{\alpha} \delta(\boldsymbol{r}),
$$

where $W_{1}, B_{1}, H_{1}, M_{1}, \mu_{1}, W_{2}, B_{2}, H_{2}, M_{2}, \mu_{2}, t_{0}, x_{0}$ and $\alpha$ are the 13 Gogny interaction parameters, and $P_{\tau}$ is the isospin exchange operator. By using Hartree-Fock approach, we explicitly write down its single-nucleon potential

$$
\begin{aligned}
U_{\tau}(\rho, \delta, k) & =\rho \sum_{i=1}^{2} \pi^{\frac{3}{2}} \mu_{i}^{3}\left(W_{i}+\frac{B_{i}}{2}\right)-\rho_{\tau} \sum_{i=1}^{2} \pi^{\frac{3}{2}} \mu_{i}^{3}\left(H_{i}+\frac{M_{i}}{2}\right)+t_{0} \rho^{\alpha}\left[\left(1+\frac{x_{0}}{2}\right) \rho-\left(\frac{1}{2}+x_{0}\right) \rho_{\tau}\right] \\
& +\frac{1}{8} t_{0} \alpha \rho^{\alpha+1}\left[3-\left(2 x_{0}+1\right) \delta^{2}\right]+\sum_{i=1}^{2} Z_{i}(k, \tau)\left(-W_{i}-2 B_{i}+H_{i}+2 M_{i}\right) \\
& +\sum_{i=1}^{2} Z_{i}(k,-\tau)\left(H_{i}+2 M_{i}\right)
\end{aligned}
$$

with

$$
Z_{i}(k, \tau)=\frac{1}{\sqrt{\pi} \mu_{i} k}\left[e^{-\mu_{i}^{2}\left(k+k_{F_{\tau}}\right)^{2} / 4}-e^{-\mu_{i}^{2}\left(k-k_{F_{\tau}}\right)^{2} / 4}\right]+\frac{1}{2}\left\{\operatorname{erf}\left[\mu_{i}\left(k+k_{F_{\tau}}\right) / 2\right]-\operatorname{erf}\left[\mu_{i}\left(k-k_{F_{\tau}}\right) / 2\right]\right\},
$$

where $\operatorname{erf}(x)$ is error function.

[1] B.A. Li, C.M. Ko, and W. Bauer, Int. Jour. Mod. Phys. E 7, 147 (1998).

[2] P. Danielewicz, R. Lacey, and W.G. Lynch, Science 298, 1592 (2002).

[3] J.M. Lattimer and M. Prakash, Science 304, 536 (2004); Phys. Rep. 442, 109 (2007).

[4] A.W. Steiner, M. Prakash, J.M. Lattimer, and P.J. Ellis, Phys. Rep. 411, 325 (2005).

[5] V. Baran, M. Colonna, V. Greco, and M. Di Toro, Phys. Rep. 410, 335 (2005).

[6] B.A. Li, L.W. Chen, and C.M. Ko, Phys. Rep. 464, 113 (2008).

[7] C.J. Horowitz, S.J. Pollock, P.A. Souder, and R. Michaels, Phys. Rev. C 63, 025501 (2001).
[8] T. Sil, M. Centelles, X. Viñas, and J. Piekarewicz, Phys. Rev. C 71, 045502 (2005).

[9] P.G. Krastev and B.A. Li, Phys. Rev. C 76, 055804 (2007).

[10] D.H. Wen, B.A. Li, and L.W. Chen, Phys. Rev. Lett. 103, 211102 (2009).

[11] B.A. Brown, Phys. Rev. Lett. 85, 5296 (2000); S. Typel and B.A. Brown, Phys. Rev. C 64, 027302 (2001).

[12] C.J. Horowitz and J. Piekarewicz, Phys. Rev. Lett. 86, 5647 (2001).

[13] R.J. Furnstahl, Nucl. Phys. A706, 85 (2002).

[14] A.E.L. Dieperink et al., Phys. Rev. C 68, 064307 (2003).

[15] L.W. Chen, C.M. Ko, and B.A. Li, Phys. Rev. C 72, 064309 (2005). 
[16] B.G. Todd-Rutel and J. Piekarewicz, Phys. Rev. Lett 95, 122501 (2005).

[17] M. Centelles, X. Roca-Maza, X. Viñas, and M. Warda, Phys. Rev. Lett 102, 122502 (2009); M. Warda, X. Viñas, X. Roca-Maza, and M. Centelles, Phys. Rev. C 80, 024316 (2009).

[18] F. Sammarruca and P. Liu, Phys. Rev. C 79, 057301 (2009).

[19] L.W. Chen, C.M. Ko, B.A. Li, and J. Xu, Phys. Rev. C 82, 024321 (2010).

[20] X. Roca-Maza, M. Centelles, X. Viñas, and M. Warda, Phys. Rev. Lett 106, 252501 (2011).

[21] K. Oyamatsu, K. Iida, and H. Koura, Phys. Rev. C 82, 027301 (2010).

[22] K. Oyamatsu and K. Iida, Phys. Rev. C 75, 015801 (2007).

[23] S. Kubis, Phys. Rev. C 76, 025801 (2007).

[24] J. Xu, L.W. Chen, B.A. Li, and H.R. Ma, Phys. Rev. C 79, 035802 (2009); Astrophys. J. 697, 1549 (2009).

[25] W.G. Newton, M. Gearheart, and B.A. Li, arXiv:1110.4043, Phys. Rev. C (in press).

[26] W.G. Newton and B.A. Li, Phys. Rev. C 80, 065809 (2009).

[27] P.G. Krastev, B.A. Li, and A. Worley, Phys. Lett. B668, 1 (2008).

[28] D.H. Wen, B.A. Li, and P.G. Krastev, Phys. Rev. C 80, $025801(2009)$

[29] W.K. Lin, B.A. Li, J. Xu, C.M. Ko, and D.H. Wen, Phys. Rev. C 83, 045802 (2011).

[30] M. Gearheart, W.G. Newton, J. Hooker, and B.A. Li, MNRAS 418, 2343 (2011) arXiv:1106.4875.

[31] D.H. Wen, W.G. Newton, and B.A. Li, arXiv:1110.5985, Phys. Rev. C (in press).

[32] M.I. Krivoruchenko, F. Šimkovic, and A. Faessler, Phys. Rev. D79, 125023 (2009).

[33] D.R. Zhang, P.L. Yin, W. Wang, Q.C. Wang, and W.Z. Jiang, Phys. Rev. C 83, 035801 (2011).

[34] D.H. Wen, B.A. Li, and L.W. Chen, arXiv:1101.1504.

[35] H. Zheng and L.W. Chen, arXiv:1111.0883, Phys. Rev. $\mathrm{D}$ (in press).

[36] L.W. Chen, C.M. Ko, and B.A. Li, Phys. Rev. Lett. 94, 032701 (2005); B.A. Li and L.W. Chen, Phys. Rev. C 72, 064611 (2005).

[37] M.B. Tsang, Y. Zhang, P. Danielewicz, M. Famiano, Z. Li, W. G. Lynch, and A. W. Steiner, Phys. Rev. Lett. 102, 122701 (2009).

[38] C. Xu, B.A. Li, and L.W. Chen, Phys. Rev. C 82, 054607 (2010).

[39] M.B. Tsang, Z. Chajecki, D. Coupland, P. Danielewicz, F. Famiano, R. Hodges, M. Kilburn, F. Lu, W.G. Lynch, J. Winkelbauer, M. Youngs, Y.X. Zhang, Prog. Part. Nucl. Phys. 66, 400 (2011).

[40] L.W. Chen, Phys. Rev. C 83, 044308 (2011).

[41] W.G. Newton, M. Gearheart, J. Hooker, and B.A. Li, arXiv:1112.2018.

[42] M.B. Tsang et al., Phys. Rev. Lett. 92, 062701 (2004).

[43] M.A. Famiano et al., Phys. Rev. Lett. 97, 052701 (2006).

[44] D.V. Shetty, S.J. Yennello, and G.A. Souliotis, Phys. Rev. C 76, 024606 (2007).

[45] W.D. Myers and W.J. Swiatecki, Nucl. Phys. A 601, 141 (1996).

[46] M. Liu, N. Wang, Z.X. Li, and F.S. Zhang, Phys. Rev. C 82, 064306 (2010).
[47] P. Danielewicz and J. Lee, Nucl. Phys. A 818, 36 (2009).

[48] A. Klimkiewicz et al., Phys. Rev. C 76, 051603(R) (2007).

[49] A. Carbone et al., Phys. Rev. C 81, 041301(R) (2010).

[50] L. Trippa, G. Coló, and E. Vigezzi, Phys. Rev. C 77, 061304 (2008).

[51] L.G. Cao and Z.Y. Ma, Chin. Phys. Lett. 25, 1625 (2008).

[52] C. Xu, B.A. Li, L.W. Chen, and C.M. Ko, Nucl. Phys. A865, 1 (2011).

[53] C. Xu and B.A. Li, Phys. Rev. C 81, 064612 (2010).

[54] N.M. Hugenholtz and L. Van Hove, Physica 24, 363 (1958).

[55] L. Satpathy, V.S. Uma Maheswari, and R.C. Nayak, Phys. Rep. 319, 85 (1999).

[56] A.M. Lane, Nucl. Phys. 35, 676 (1962).

[57] B.A. Li , Phys. Rev. C 69, 064602 (2004).

[58] A.J. Koning and J.P. Delaroche, Nucl. Phys. A713, 231 (2003).

[59] X.H. Li and L.W. Chen, Nucl. Phys. A874, 62 (2012) arXiv:1108.4858.

[60] J. Bartel et al., Nucl. Phys. A386, 79 (1982).

[61] E. Chabanat, P. Bonche, P, Haensel, J. Meyer, and R. Schaeffer, Nucl. Phys. A635, 231 (1998); Nucl. Phys. A643, 441 (1998)

[62] J. Decharge and D. Gogny, Phys. Rev. C 21, 1568 (1980).

[63] J.F. Berger, M. Girod, and D. Gogny, Comp. Phys. Comm. 63, 365 (1991).

[64] F. Chappert, M. Girod, and S. Hilaire, Phys. Lett. B668, 420 (2008).

[65] S. Goriely, S. Hilaire, M. Girod, and S. Peru, Phys. Rev. Lett. 102, 242501 (2009).

[66] D. Vautherin and D.M.Brink, Phys. Rev. C 5, 626 (1972).

[67] J. Friedrich and P.-G. Reinhard, Phys. Rev. C 33, 335 (1986).

[68] M.J. Giannoni and P. Quentin, Phys. Rev. C 21, 2076 (1980).

[69] J.M. Pearson and R.C. Nayak, Nucl. Phys. A668, 163 (2000).

[70] J.M. Pearson and S. Goriely, Phys. Rev. C 64, 027301 (2001).

[71] J. Margueron, J. Navarro, and N. Van Giai, Phys. Rev. C 66, 014303 (2002).

[72] S. Goriely, M. Samyn, M. Bender, and J.M. Pearson, Phys. Rev. C 68, 054325 (2003).

[73] F. Tondeur, S. Goriely, J.M. Pearson, and M. Onsi, Phys. Rev. C 62, 024308 (2000).

[74] M. Samyn et al., Nucl. Phys. A700, 142 (2002).

[75] S. Goriely et al., Phys. Rev. C 66, 024326 (2002).

[76] S. Goriely, M. Pearson, and F. Tondeur, Nucl. Phys. A688, 349 (2001).

[77] S. Goriely, F. Tondeur, and J.M. Pearson, At. Data Nucl. Data Tables 77, 311 (2001).

[78] M. Beiner, H. Flocard, N. van Giai, and P. Quentin, Nucl. Phys. A238, 29 (1975).

[79] M. Samyn, S. Goriely, M. Bender, and J.M. Pearson, Phys. Rev. C 70, 044309 (2004).

[80] A. K. Dutta et al., Nucl. Phys. A454, 374 (1986).

[81] B.A. Brown and W.A. Richter, Phys. Rev. C 58, 2099 (1998).

[82] F. Tondeur, M. Brack, M. Farine, and J.M. Pearson, Nucl. Phys. A420, 297 (1984). 
[83] M. Rayet, M. Arnould, G. Paulus, and F. Tondeur, Astron. Astrophys. 116, 183 (1982).

[84] C.J. Pethick, D.G. Ravenhall, and C.P. Lorenz, Nucl. Phys. A584, 675 (1995).

[85] S. Goriely and J.M. Pearson. Phys. Rev. C 77, 031301 (2008).

[86] S. Goriely, M. Samyn, and J. Pearson, Nucl. Phys. A773, 279 (2006).

[87] N. Van Giai and H. Sagawa, Phys. Lett. B106, 379 (1981).

[88] E. Chabanat, Interactions effectives pour des conditions extremes d'isospin, Ph.D. thesis, University Claude Bernard Lyon-1, Lyon, France, 1995.

[89] N. Chamel, S. Goriely, and J.M. Pearson, Nucl. Phys. A812, 72 (2008).

[90] S. Goriely, N. Chamel, and J.M. Pearson, Phys. Rev. Lett. 102, 152503 (2009).

[91] B.K. Agrawal, S. Shlomo, and V.K. Au, Phys. Rev. C 72, 014310 (2005).

[92] S. Goriely, M. Samyn, and J.M. Pearson, Phys. Rev. C 75, 064312 (2007).

[93] H. Krivine, J. Treiner, and O. Bohigas, Nucl. Phys. A336, 155 (1980).

[94] M.M. Sharma, G.A. Lalazissis, J. König, and P. Ring, Phys. Rev. Lett. 74, 3744 (1995).

[95] W. Nazarewicz et al., Phys. Rev. C 53, 740 (1996).

[96] P.-G. Reinhard and H. Flocard, Nucl. Phys. A584, 467 (1995).

[97] L.G. Cao, U. Lombardo, C.W. Shen, and N. Van Giai, Phys. Rev. C 73, 014313 (2006).

[98] P.-G. Reinhard et al., Phys. Rev. C 60, 014316 (1999).

[99] L. Bennour et al., Phys. Rev. C 40, 2834 (1989).

[100] H.S. Kohler, Nucl. Phys. A258, 301 (1976).

[101] C.M. Ko, H.C. Pauli, M. Brack, and G.E. Brown, Nucl. Phys. A236, 269 (1974).

[102] B.K. Agrawal, S. Shlomo, and V.K. Au, Phys. Rev. C 68, 031304 (2003).

[103] L.W. Chen, C.M. Ko, and B.A. Li, Phys. Rev. C 72, 064606 (2005).

[104] Z.H. Li, L.W. Chen, C.M. Ko, B.A. Li, and H.R. Ma, Phys. Rev. C 74, 044613 (2006).

[105] D.P. Murdock and C.J. Horowitz, Phys. Rev. C 35, 1442
(1987).

[106] J.A. McNeil, L. Ray, and S.J. Wallace, Phys. Rev. C 27, 2123 (1983).

[107] E.N.E. van Dalen, C. Fuchs, and A. Faessler, Phys. Rev. C 72, 065803 (2005).

[108] W. Zuo, U. Lombardo, H.-J. Schulze, and Z.H. Li, Phys. Rev. C 74, 014317 (2006).

[109] C.B. Das, S. Das Gupta, C. Gale, and B.A. Li, Phys. Rev. C 67, 034611 (2003).

[110] B.A. Li, C. B. Das, S. Das Gupta, and C. Gale, Phys. Rev. C 69, 011603(R) (2004); Nucl. Phys. A735, 563 (2004).

[111] L.W. Chen, C.M. Ko, and B.A. Li, Phys. Rev. C 69, $054606(2004)$

[112] B.A. Li, G.C. Yong, and W. Zuo, Phys. Rev. C 71, 014608 (2005).

[113] B.A. Li, G.C. Yong, and W. Zuo, Phys. Rev. C 71, 044604 (2005).

[114] B.A. Li, L.W. Chen, G.C. Yong, and W. Zuo, Phys. Lett. B634, 378 (2006).

[115] G.C. Yong, B.A. Li, L.W. Chen, and W. Zuo, Phys. Rev. C 73, 034603 (2006).

[116] G.C. Yong, B.A. Li, and L.W. Chen, Phys. Rev. C 74, 064617 (2006).

[117] G.C. Yong, B.A. Li, and L.W. Chen, Phys. Lett. B650, 344 (2007).

[118] J. Xu, L.W. Chen, B.A. Li, and H.R. Ma, Phys. Rev. C 75, 014607 (2007).

[119] J. Xu, L.W. Chen, B.A. Li, and H.R. Ma, Phys. Lett. B650, 348 (2007).

[120] J. Xu, L.W. Chen, C.M. Ko, and B.A. Li, Phys. Rev. C 81, 055803 (2010).

[121] J. Xu and C.M. Ko, Phys. Rev. C 82, 044311 (2010).

[122] L.W. Chen, C.M. Ko, and B.A. Li, Phys. Rev. C 76, 054316 (2007).

[123] L.W. Chen, B.J. Cai, C.M. Ko, B.A. Li, C. Shen, and J. Xu, Phys. Rev. C 80, 014322 (2009).

[124] E. Chabanat, P. Bonche, P, Haensel, J. Meyer, and R. Schaeffer, Nucl. Phys. A627, 710 (1997).

[125] D. Gogny and R. Padjen, Nucl. Phys. A293, 365 (1977). 